\title{
Electroacupuncture Pretreatment Attenuates Cerebral Ischemia-Reperfusion Injury through Transient Receptor Potential Vanilloid 1-Mediated Anti-Apoptosis via Inhibiting NF-kB Signaling Pathway
}

\section{Nina Yin}

Hubei University of Chinese Medicine

\section{Man Long}

Hubei University of Chinese Medicine

\section{Zhigang Wang}

Hubei University of Chinese Medicine

\section{Luyao Shao}

Hubei University of Chinese Medicine

Jing Bi

Hubei University of Chinese Medicine

Zebin Chen ( $\nabla$ chenzebin-hbtcm@outlook.com )

Hubei University of Chinese Medicine https://orcid.org/0000-0003-0054-3665

\section{Research Article}

Keywords: electroacupuncture, TRPV1, apoptosis, cerebral ischemia-reperfusion injury, NF-kB signaling pathway, ischemic stroke.

Posted Date: June 30th, 2021

DOI: https://doi.org/10.21203/rs.3.rs-657177/v1

License: (c) (i) This work is licensed under a Creative Commons Attribution 4.0 International License. Read Full License 


\section{Abstract}

Our previous study showed that electroacupuncture (EA) pretreatment elicited protective effect on cerebral ischemia-reperfusion injury in rats, at least partly, which was associated with transient receptor potential vanilloid 1 (TRPV1)-regulated anti-oxidant stress and anti-inflammation. In this study, we further investigated the possible contribution of TRPV1-mediated anti-apoptosis in EA pretreatment-evoked neuroprotection. After EA pretreatment at Baihui (GV20), bilateral Shenshu (BL23) and Sanyinjiao (SP6) acupoints, transient focal cerebral ischemia was induced by middle cerebral artery occlusion (MCAO) for $2 \mathrm{~h}$ followed by reperfusion for $6 \mathrm{~h}$ in rats. Then, infarct volume, nerve cell injury, neuronal apoptosis, NFKB signaling activation, and expression of TRPV1 were evaluated by TTC staining, hematoxylin-eosin staining, transmission electron microscopy, immunochemistry, immunofluorescence, and Western blot, respectively. The presented data showed that EA pretreatment significantly reduced infarct volume, relieved nerve cell injury, decreased the expression of pro-apoptotic proteins Bax and cleaved caspase-3, increased the level of anti-apoptotic protein Bcl-2, inhibited NF-KB (p65) transcriptional activity, and curbed TRPV1 expression in MCAO rats. By contrast, enhancement of TRPV1 expression accompanying capsaicin application, the specific TRPV1 agonists, markedly accelerated nerve cell damage, aggravated neuronal apoptosis, prompted nuclear translocation of NF-KB (p65), resulting in the reversion of EA pretreatment-evoked neuroprotective effect in MCAO rats. Thus, we conclude that EA pretreatmentinduced downregulation of neuronal TRPV1 expression plays an anti-apoptosis role through inhibiting NF-kB signaling pathway, thereby protecting MCAO rats from cerebral ischemia-reperfusion injury.

\section{Introduction}

Stroke, a sudden circulatory disturbance of cerebrovascular blood flow, is defined as a neurological disease with high morbidity, disability, and mortality. Generally, stroke is broadly divided into two main categories: ischemic stroke and hemorrhagic stroke, and the former accounts for about $80 \%$ of stroke cases [1]. Accompanying occlusion caused by a thrombus or embolus in cerebrovascular circulation, ischemic stroke always occurs due to decreased or blocked blood flow to brain tissues [2]. Although reconstruction or enhancement of blood flow in the ischemic region is considered to restore cerebrovascular occlusion, the recovery of blood flow after a certain period of time might aggravate local ischemia thereby causing secondary damage to neuronal function, which is called cerebral ischemiareperfusion injury (CIRI) [3]. So far, only thrombolysis treatment has received a clinical approval and is recommended to apply in ischemic stroke, including recombinant tissue-plasminogen activator (r-tPA) and mechanical thrombectomy [4]. Therefore, increasing efforts have been focused on developing new therapeutic strategies, including numerous types of pretreatment measurements, to prevent or limit CIRI so that more stroke patients can benefit from its clinical applications.

During CIRI associated with ischemic stroke, a series of detrimental cascade events are aroused and eventually lead to neuronal death, such as mitochondrial dysfunction, overproduction of free radicals, $\mathrm{Ca}^{2+}$ overload, excitotoxicity, oxidative stress, inflammation, ion imbalance, and apoptosis [5]. Of note, increasing evidence indicates that apoptosis, a genetically programmed cell death process, plays an 
important role in the progression of CIRI based on the interconnection of cascades reactions [6]. The extrinsic apoptotic pathway is associated with the activation of death receptors that bind specific ligands and transmit apoptotic signals. However, the intrinsic apoptotic pathway is triggered by intracellular stimuli such as DNA damage, hypoxia, and oxidative stress, which consequently lead to outer mitochondrial membrane permeabilization, contributing to cellular degradation of apoptosis [7]. Inhibition of apoptosis, including the downregulation of pro-apoptosis protein caspases 3, 6, 9 and Bax as well as the upregulation of anti-apoptosis protein $\mathrm{Bcl}-2$, prevents neuronal damage and death, therefore regulation of apoptosis represents a valuable research direction for stroke rehabilitation [8].

As a branch of traditional Chinese medicine, acupuncture has been widely accepted in Eastern and Western countries with incomparable advantages, including simple operation, convenience, affordable cost, good repeatability, efficacy, safe, and no side effects [9]. Electroacupuncture (EA) pretreatment, application in advance through inserting a metal needle at specific acupoints on the body surface with stimulation delivery via electric pulses, has been reported to be effective for treating CIRI or ischemic stroke through different mechanisms, such as inhibition of inflammation, suppression of oxidant stress, regulation of autophagy, attenuation of neuronal apoptosis $[10,11]$. Our recent study showed that EA pretreatment elicited neuroprotective effect on CIRI in rats, the mechanism of which was related to transient receptor potential vanilloid 1 (TRPV1)-regulated anti-oxidant stress and anti-inflammation [12]. Previous studies also indicated the involvement of TRPV1 channels and apoptosis in CIRI or ischemic stroke [13-15]. However, whether TRPV1-mediated anti-apoptosis contributes to EA pretreatment-elicited neuroprotection is still unclear.

In this study, we firstly investigated the effects of EA pretreatment on neuronal apoptosis and TRPV1 expression in MCAO rats, and further clarified the relationship between EA pretreatment-evoked neuroprotection and TRPV1-mediated anti-apoptosis. Our results provide a new evidence that EA pretreatment exerts its beneficial action to improve cerebral ischemia-reperfusion injury in MCAO rats, at least partly, attributing to TRPV1-mediated anti-apoptosis through inhibiting NF-KB signaling pathway.

\section{Materials And Methods}

\section{Animals}

Six-week-old male Sprague-Dawley rats $(220 \pm 20 \mathrm{~g})$ were purchased from Hubei Research Center of Laboratory Animals (Wuhan, China) (No.: 211002300044504).

\section{EA pretreatment}

A Han's Acupoint Nerve Stimulator (HANS-200A, Nanjing Jisheng Medical Technology Co., Ltd., Nanjing, China) was applied to "Baihui" (GV20, located at the intersection of sagittal midline and the line linking two rat ears), bilateral "Shenshu" (BL23, on both sides of the 2nd lumbar vertebra, and the distance was $6 \mathrm{~mm}$ from the dorsmedian) and "Sanyinjiao" (SP6, the tip of the medial malleolus of the hind limb is straight up 10mm) acupoints (Fig. 1a and b). BL23 and SP6 acupoints on the same side 
were used together as pairs, and a shallow needle was prickled at $0.5 \mathrm{~cm}$ near Baihui point as an auxiliary electrode to form a pair with GV20 acupoint. The needles (diameter $0.25 \mathrm{~mm}$ and length $25 \mathrm{~mm}$ ) (Beijing Zhongyan Taihe Medical Instrument Co., Ltd., Beijing, China) were inserted into the skin. Then, a dilatational wave at $2 / 100 \mathrm{~Hz}$ and $1 \mathrm{~mA}$ with electrical stimulation was used for $10 \mathrm{~min}$, and kept the needles alone without electrical stimulation for $5 \mathrm{~min}$. The process was continually repeated 4 times for 1 $\mathrm{h}$ in total as EA pretreatment.

\section{Middle cerebral artery occlusion (MCAO) model}

Briefly, rats received intraperitoneal anesthesia with $5 \%$ chloral hydrate $(0.6 \mathrm{~mL} / 100 \mathrm{~g})$. A monofilament nylon suture with a rounded tip coated with silicone rubber (2032/2432A5, Beijing Cinontech Co., Ltd., Beijing, China) was inserted into the internal carotid artery $18-20 \mathrm{~mm}$ to block the blood flow. When ischemia was reached for $2 \mathrm{~h}$, the middle cerebral artery was re-perfused by withdrawing the silicone rubber coated monofilament to induce the MCAO model of focal cerebral ischemia-reperfusion injury in rats.

\section{Experimental protocols}

\section{Experiment I}

72 Rats were randomly distributed to four groups ( $n=18$ for each group): middle cerebral artery occlusion model group (MCAO), sham-operation group with surgery but no MCAO (Sham), EA pretreatedMCAO group (EA + MCAO), and sham EA pretreated-MCAO group (N-EA + MCAO). For sham EA pretreatment $(\mathrm{N}-\mathrm{EA})$, the needles were inserted vertically into five acupoints only about 2-3 $\mathrm{mm}$ deep under the skin without electricity output, but procedures, electrode placements, and other treatment settings were the same as EA pretreatment. The sensations of "de qi" were not achieved in the sham EA pretreatment. As shown in Fig. 1C, rats were pretreated with EA stimulation at GV20, bilateral BL23 and SP6 acupoints for $1 \mathrm{~h}$. Next, the rats were yield to unilateral right middle cerebral artery occlusion for $2 \mathrm{~h}$, and then re-perfused for $6 \mathrm{~h}$. Lastly, rats were sacrificed to collect the samples.

\section{Experiment II}

60 Rats were randomly divided into four groups ( $n=15$ for each group): MCAO, EA + MCAO, capsaicin administration before MCAO model (Capsaicin + MCAO), and capsaicin administration before EA pretreated-MCAO group (Capsaicin + EA + MCAO). According to previous publication [16], capsaicin (the specific TRPV1 agonists, $0.2 \mathrm{mg} / \mathrm{kg}$ via s.c.) (S1990, Selleckchem, Houston, TX, USA) in advance was applied $2 \mathrm{~h}$ before EA pretreatment (Fig. 7a). Then, EA stimulation at GV20, bilateral BL23 and SP6 acupoints for $1 \mathrm{~h}$ prior to MCAO model. Lastly, rats were sacrificed to collect the samples.

\section{Measurement of infarct volume}

After rats were sacrificed, the brains were collected and infarct volume was measured according to previous description [17]. The brain tissue was sectioned into six coronal blocks with an approximate 
thickness of $2 \mathrm{~mm}$. Then, the sections were stained with 1\% 2,3,5-triphenyltetrazolium chloride (TTC) (T8877, Sigma-Aldrich, St. Louis, MO, USA) for 20 min and immersed in 4\% paraformaldehyde overnight. Normal area presented red and infarct area kept white. The percentage of infarct volumes were calculated following the formula: Infarct volumes $(\%)=\left(V_{c}-V_{L}\right) / V_{c} \times 100 \%$

$V_{c}=$ volumes of normal gray matter in the control hemisphere

$V_{L}=$ volumes of normal gray matter in the s lesioned hemisphere

\section{Histological analysis}

At the end of the experiment, brain tissues of rats were removed and fixed in $4 \%$ paraformaldehyde. Fixed tissues were embedded in paraffin and serially sectioned to a thickness of $5 \mu \mathrm{m}$. Then, the sections were stained with hematoxylin and eosin (H\&E) and examined for histological analysis. A minimum of three sections per animal experimentation was examined, and five visual fields of each sample were randomly selected to observe nerve cell injury in a blinded manner.

\section{Transmission electron microscopy}

Fresh brain tissues were taken from the ischemic cerebral hippocampus, cut into $1 \mathrm{~mm}^{3}$ size cubes and fixed in $2.5 \%$ glutaraldehyde for $24 \mathrm{~h}$. Next, the samples were fixed in $1 \%$ osmium tetroxide for $2 \mathrm{~h}$ and dehydrated in graded ethanol and embedded in araldite. Then, the sections were cut at $50 \mathrm{~nm}$ and stained with uranyl acetate and lead citrate. Finally, the ultrastructure of neurons and mitochondria were observed with Tecnai G2 20 200kV transmission electron microscope (FEl/Philips Electron Optics, Eindhoven, Netherlands).

\section{Immunohistochemistry}

For immunohistochemical analysis, the paraffin sections of brain tissues were deparaffinized, blocked with $5 \%$ BSA, and treated with rabbit anti-rat Bax (ab32503), Bcl2 (ab194583) (Abcam, Cambridge, Massachusetts, USA), cleaved caspase-3 (\#9661) (Cell Signaling Technology Inc., Beverly, Massachusetts, USA) and TRPV1 (PA5-77317) (Invitrogen, Carlsbad, CA, USA) at $37^{\circ} \mathrm{C}$ for $2 \mathrm{~h}$, separately. The sections were incubated with second antibody (ab6720, Abcam) at $37^{\circ} \mathrm{C}$ for $30 \mathrm{~min}$. Then, SABC complex was added and the slides were stained with DAB solution. Finally, the slides were observed with a microscope (Olympus BX40, Tokyo, Japan). Under $\times 400$ magnification, the morphometric examination was performed in a blinded manner by two independent investigators. Cells with brown granule staining of the membrane/cytoplasm were considered positive. For each section, five visual fields were chosen at random and mean number of the positive cells were represented.

\section{Western blotting}

Rat hippocampus were collected and extracted using ice-cold RIPA lysis buffer (P0013B, Beyotime, Shang, China). Protein concentration was determined with a BCA Protein Assay Kit (23227, ThermoFisher 
Scientific, San Jose, CA, USA). Equivalent amounts of protein $(25 \mu \mathrm{g})$ were separated by $10 \%$ sodium dodecyl sulfate-polyacrylamide gel electrophoresis. Then, it was transferred onto PVDF membranes. The membranes were blocked with $5 \%$ skim milk for $2 \mathrm{~h}$ and followed by incubation overnight at $4{ }^{\circ} \mathrm{C}$ with primary rabbit anti-rat antibodies against Bax (ab32503), Bcl-2 (ab194583), Histone H3 (ab176842) (Abcam), cleaved caspase-3 (\#9661), IKBa (\#4812), p-IкBa (\#2859), NF-кB (p65) (\#8242), p-NF-kB (p65) (\#3033) (Cell Signaling Technology Inc.), GAPDH (BM3874) (Boster Biological Technology Co., Ltd, Wuhan, China), and TRPV1 (PA5-77317) (Invitrogen). Then, the membranes were held with HRPconjugated goat anti-rabbit antibody (\#7074) (Cell Signaling Technology Inc.). The protein intensity was detected by the ChemiDoc ${ }^{\text {TM }}$ XRS+ system (Bio-Rad Laboratories, Inc., Hercules, CA, USA) and analyzed with ImageJ software (National Institutes of Health, Bethesda, MD, USA). Sample loading was normalized by quantity of GAPDH or Histone $\mathrm{H} 3$ detected parallel.

\section{Immunofluorescence}

For dual immunofluorescence labeling, the slides of brain tissues were rinsed in $0.1 \mathrm{M}$ PBS, blocked for 1 $\mathrm{h}$ with $2 \% \mathrm{BSA}$ and then incubated with the following primary antibodies at $4^{\circ} \mathrm{C}$ overnight: rabbit antiTRPV1(PA5-77317) and anti-NeuN (PA5-78499) (Invitrogen). For labeling, the primary antibodies were detected with corresponding secondary antibodies: donkey anti-rabbit IgG conjugated with Alexa Fluor ${ }^{\circledR} 488$ (711-545-152) or Alexa Fluor ${ }^{\circledR} 594$ (711-585-152) (Jackson ImmunoResearch, West Grove, PA, USA). Lastly, the sections were stained with DAPI (AR1177) (Boster Biological Technology, Wuhan, China) and examined under a fluorescence microscope (Olympus IX73, Tokyo, Japan).

\section{Statistical analysis}

Results were presented as the mean \pm SD. Data were analyzed with SPSS 19.0 software (SPSS Inc., Chicago, IL, USA). Differences between two groups were evaluated by the Mann-Whitney U test, and multiple comparisons were analyzed using the Kruskal-Wallis test. When result of the Kruskal-Wallis test was significant, a Dunn test was performed for post hoc analysis. A value of $P<0.05$ was considered statistically significant.

\section{Results}

\section{EA pretreatment prevents rats from cerebral ischemia-reperfusion injury}

In this study, GV20, bilateral BL23 and SP6 acupoints were used (Fig. 1a and b) according to the experimental progress presented in Fig. 1c (Experiment I). Compared with MCAO group, infarct regions (white) (Fig. 1d) and the percentage of infarct volume were significantly decreased in EA + MCAO group (Fig. 1e). Simultaneously, EA pretreatment relieved nerve cell injury in the hippocampus of MCAO rats, such as reactive hyperplasia of glial cells, nuclear pyknosis, and even cell death (Fig. 2a).

Under transmission electron microscope, neuronal nucleus in the hippocampus turned into smaller and transformed into pyknosis, deformation and even lysis in MCAO group. Additionally, the mitochondrial ultrastructure of neurons presented degenerative signs, such as vacuolation and swelling (Fig. 2b). EA 
pretreatment significantly relieved neurons and mitochondria injury accompanying alleviation of nerve cell damage as described above (Fig. 2), suggesting neuroprotective activity of EA pretreatment in MCAO rats.

\section{EA pretreatment inhibits hippocampal neuronal apoptosis}

Next, we assessed the expression of apoptosis-related proteins via immunohistochemical staining, including Bax, Bcl-2, and cleaved caspase-3. As shown in Fig. 3a, the numbers of Bax- and cleaved caspase-3-positive cells were both markedly decreased in EA + MCAO group in comparison with MCAO group, while Bcl-2-positive cells were increased in MCAO rats with EA application in advance (Fig. 3b-d). The inhibitive effect of EA pretreatment on apoptosis-related protein expression was also confirmed by Western blot analysis (Fig. 4a and c), and the similar trends, i.e., reduction of Bax/Bcl-2 and cleaved caspase-3/GAPDH ration in EA + MCAO group compared with MCAO group, was presented in Fig. $4 \mathrm{~b}$ and d.

\section{EA pretreatment suppresses NF-KB signaling activation in MCAO rats}

To explore molecular mechanism of the anti-apoptosis effect of EA pretreatment in MCAO rats, we further investigated the protein expression associated with NF-KB signaling pathway, including IKBa, $\mathrm{p}$ IKBa, NF-KB (p65), and p-NF-KB (p65). Compared with Sham group, increasing cytosolic phosphorylation of IKBa and nuclear translocation of p-NF-KB (p65) as well as decreasing cytosolic NF-kB (p65) subunit were showed in MCAO rats (Fig. $5 \mathrm{a}$ and $\mathrm{c}$ ). In contrast to the MCAO group, relative protein expression of $\mathrm{p}-\mathrm{I} \mathrm{KBa} / \mathrm{IKBa}$ and $\mathrm{p}-\mathrm{NF}-\mathrm{kB}(\mathrm{p} 65) / \mathrm{NF}-\mathrm{kB}(\mathrm{p} 65)$ were both reduced in EA + MCAO group (Fig. $5 b$ and $d)$.

\section{EA pretreatment reduces TRPV1 expression in the hippocampus of MCAO rats}

As shown in Fig. 6a, immunohistochemistry showed the number of TRPV1-positive cells was upregulated in MCAO group compared with that in Sham group (Fig. 6b). EA pretreatment in MCAO rats reduced the TRPV1-positive cells and downregulated protein expression of TRPV1 in comparison with MCAO group (Fig. 6a-d). Furthermore, double immunofluorescence labeling indicated colocalization expression of TRPV1 and NeuN in the hippocampal neurons. In comparison with the MCAO group, EA pretreatment decreased the colocalization of TRPV1 and NeuN (Fig. 7), accompanying the increment of TRPV1positive cells and decrement of NeuN-positive cells. Colocalization analysis suggests that functional effects of TRPV1 contribute more to EA pretreatment-evoked neuroprotection relative to recovery of the number of neurons.

\section{Capsaicin abrogates EA pretreatment-evoked neuroprotection in MCAO rats}

To further confirm the role of TRPV1 in the neuroprotective benefits of EA pretreatment, capsaicin, the specific TRPV1 agonists, was used in MCAO rats according to the schematic diagram of Experiment II described in Fig. 8a. As shown in Fig. 8b and c, capsaicin application obviously promoted TRPV1 expression at the protein level. Importantly, capsaicin significantly abrogated the inhibitive effect of EA 
pretreatment on TRPV1 expression in MCAO rats (Fig. 8d and e). Capsaicin administration reversed the neuroprotective effect of EA pretreatment in MCAO rats, which was emphasized by the increment of infarct regions and the augmentation of infarct volume (Fig. $8 \mathrm{f}$ and g).

\section{Capsaicin abolishes anti-apoptosis activity of EA pretreatment in MCAO rats}

Following early application of capsaicin, the protective effect of EA pretreatment on nerve cell injury of the hippocampus in MCAO rats was abolished, such as increment of reactive hyperplasia of glial cells, development of cells with nuclear pyknosis, and appearance of dead cells (Fig. 9a). Compared with EA + MCAO group, the numbers of Bax- and cleaved caspase-3-positive cells were both increased as well as Bcl-2-positive cells decreased in capsaicin + EA + MCAO group (Fig. 9b-e). Simultaneously, capsaicin significantly raised the relative expression of $\mathrm{Bax} / \mathrm{Bcl}-2$ and cleaved caspase-3/GAPDH in MCAO rats with EA pretreatment at the protein level (Fig. 10a-d).

\section{Capsaicin revoked the suppressive effect of EA pretreatment on NF-KB signaling activation in MCAO rats}

Lastly, we examined NF-KB signaling activation in MCAO rats in response to capsaicin application. Compared with those in EA + MCAO group, phosphorylation of cytoplasmic IKBa and nuclear translocation of p-NF-kB (p65) (Fig. 11a and c), as well as relative protein expression of $\mathrm{p}-\mathrm{IkBa} / \mathrm{lkBa}$ and p-NF-kB (p65)/NF-KB (p65), were both enhanced in capsaicin + EA + MCAO group (Fig. 11b and d). These findings highlight the involvement of TRPV1-mediated anti-apoptosis in EA pretreatment-evoked neuroprotection via inhibiting NF-KB signaling activation.

\section{Discussion}

Prior to cerebral ischemia, certain "pretreatment" stimulation can develop resistance or tolerance to protect against subsequent injury such as promoting cell survival, which highlights the viewpoint of disease prevention in modern medicine [18]. EA pretreatment reflects the theory of "Zhi wei bing" (treating before sick) in traditional Chinese medicine, wherefore it may be clinically applicable for prevention [19], and not just as a complementary therapy against ischemic stroke [20]. According to our current knowledge, the concept of "EA pretreatment" was first proposed in 2003, indicating that pretreatment with repeated EA at Baihui acupoint (GV20) attenuated transient focal cerebral ischemic injury in MCAO rats [21]. Besides GV20 [22], EA pretreatment in CIRI or ischemic stroke is always associated with acupuncture prescriptions of single acupoint or acupoints combination, such as Shuigou (GV26) [23], Sanyinjiao (SP6), Neiguan (PC6) [24], Dazhui (GV14) [25], Zusanli (ST36), and Quchi (LI11) [26], etc. Although the neuroprotective effect of EA pretreatment has been widely reported and confirmed, there is currently no uniform standard for the selection and combination of acupoints because of the complicated issues, such as acupoint indications, clinical syndrome differentiation, and individualization of treatment. Under the guide of "Biao ben pei xue" acupuncture prescription, in combination of clinical syndrome and acupoint indications, two or more acupoints are combined to enhance the collaborative effects of acupoints in order to achieve specific efficacy and improve clinical outcomes [27]. In this study, GV20, bilateral BL23 and SP6 acupoints were selected and used according to our previous publication [12]. EA 
pretreatment reduced infarct volume, relieved neuronal and mitochondrial damage, and protects hippocampal neuronal from CIRI (Figs. 1 and 2), which further emphasized the neuroprotective activity of EA pretreatment in CIRI and ischemic stroke.

During ischemic stroke, molecular cascades that contribute to neuronal cell death are diverse and have been subdivided into three main types, i.e., necrosis, apoptosis, and autophagy [28, 29]. Due to the overlap between some forms of cell death and between cell death and other processes, it is hard to clearly distinguish the difference among the three under pathological conditions. Generally, apoptosis is diagnosed based on the following characteristics, including DNA fragmentation, changes of Bcl-2 family proteins, caspase activation, or phosphatidylserine exposure [30]. Increasing evidences has shown that regulation of apoptosis plays an essential role in the neuroprotection of EA pretreatment, which usually is related to upregulation of Bcl-2/Bax and downregulation of cleaved caspase-3 [31]. Simultaneously, mediation of signaling pathway is also associated with the anti-apoptosis process of EA pretreatment in CIRI and ischemic stroke [32]. A recent study showed that EA pretreatment elicited tolerance to CIRI through inhibiting GluN2B/m-calpain/p38 MAPK proapoptotic pathway [24]. Utilizing MCAO rat model, EA pretreatment exerted the neuroprotective effects by inhibiting neuronal apoptosis and autophagy via activating PI3K/AKT/mTOR signaling pathway after ischemic stroke [33]. Other publication reported that EA pretreatment exerted neuroprotection against ischemic injury through Notch pathway-mediated upregulation of hypoxia inducible factor-1 a [34]. Consistent with previous publications, EA pretreatment increased the level of anti-apoptotic protein Bcl-2 as well as decreased the expression of pro-apoptotic protein Bax and cleaved caspase-3 (Figs. 3 and 4). Different with above reports, inhibition of NF-KB signaling activation participated in the anti-apoptosis activity of EA pretreatment (Fig. 5). Whether other signaling pathways (such as PI3K/Akt, P38 MAPK, and Notch) or transcription factors (HMGB1, HIF-1a, and CREB) get involved in the neuroprotection of EA pretreatment against ischemic stroke, further studies are needed to shed light on the details.

TRPV1 is a nonselective calcium-permeable cation channel and are mainly expressed in the hippocampus and dorsal root ganglion [35]. There is accumulating evidence indicating the potential involvement of TRPV1 channels in ischemia-reperfusion injury [36]. Ischemic stroke incurred activation of TRPV1 in brain of mice, where neurological deficits and infarct volumes in TRPV1-KO mice were lower than those in WT mice [37]. Additionally, the mRNA expression of TRPV1 was significantly increased in the brain tissues of MCAO rats [38]. Utilizing a rat model of stroke, administration of AMG9810 (a TRPV1 antagonist) significantly reduced the infarct volume and decreased the neurological deficits. However, capsaicin (a TRPV1 agonist) increased the infarct volume after the induction of permanent MCAO [39]. Collectively, these studies suggest that TRPV1 exerts a deleterious effect on brain ischemic injury. Although accumulating data indicates the effects of EA on TRPV1 in the different experimental conditions, such as pain [40-42], spared nerve injury [43] and obesity [44], few studies focused on the relationship between EA treatment/pretreatment and TRPV1 in CIRI or stroke. A previous study reported that TRPV1 receptors were significantly increased in the hippocampal CA1 areas of MCAO rats, and the phenomenon was reversed by EA treatment at GV20 [45]. EA pretreatment exerted neuroprotection in MCAO rats with CIRI through TRPV1-mediated anti-oxidant stress and anti-inflammation via inhibiting 
P38 MAPK activation [12]. In the current study, TRPV1 expression was increased in the brain of MCAO rats, and EA pretreatment reduced the increment of TRPV1 in rat hippocampus (Figs. 6 and 7).

Importantly, capsaicin significantly abrogated EA pretreatment-triggered anti-apoptosis and inhibition of NF-kB nuclear translocation (Fig. 8-11), which highlighted the involvement of TRPV1-mediated antiapoptosis and suppression of NF-KB signaling activation in EA pretreatment-evoked neuroprotection.

In conclusion, EA pretreatment at GV20, bilateral BL23 and SP6 acupoints decreased TRPV1 expression in the MCAO rats, thereby curbed NF-KB transcriptional activity and played an anti-apoptosis role, contributing to the neuroprotective effect of EA pretreatment in CIRI (Fig. 10). These findings suggest the involvement of TRPV1-mediated anti-apoptosis in EA pretreatment-evoked neuroprotection via inhibiting NF-KB signaling activation, which provide a theoretical basis for the clinical application of EA pretreatment in the prevention of cerebral ischemia-reperfusion injury or ischemic stroke.

\section{Declarations}

Acknowledgements We thank Mrs Jiaojiao Chen for her help with the sample preparation for transmission electron microscopy.

Authors' contributions N.N.Y. and Z.B.C. designed the study. N.N.Y. and M.L. performed the experiments and analyzed the data. Z.G.W. and J.B. contributed to animal feeding and preparation of MCAO model. N.N.Y. wrote the draft manuscript. L.Y.S revised the final version before submission.

Funding This study was supported by the grant from 2020 Scientific Research Project of Hubei Provincial Department of Education (No.: B2020101), and Qihuang Project of National Administration of Traditional Chinese Medicine [2018]-284 (No.: HBPCIC-2019-02, HBPCIC-2019-08).

Availability of data and material Available upon request

\section{Compliance with Ethical Standards}

Ethics approval All rats were handled in accordance with the guidelines of the Animal Care and Use Committee of Hubei University of Chinese Medicine (No.: SYXK2017-0067) and conformed to the National Institutes of Health Guidelines for Animal Research.

Consent for publication All authors have approved the manuscript and agree with its publication.

Consent to participate Not applicable

Conflict of interest The authors declare no conflicts of interest.

\section{References}


1. Grysiewicz RA, Thomas K, Pandey DK (2008) Epidemiology of ischemic and hemorrhagic stroke: incidence, prevalence, mortality, and risk factors. Neurol Clin 26(4):871-895.

https://doi.org/10.1016/j.ncl.2008.07.003

2. Pan J, Konstas AA, Bateman B, Ortolano GA, Pile-Spellman J (2007) Reperfusion injury following cerebral ischemia: pathophysiology, MR imaging, and potential therapies. Neuroradiology 49(2):93102. https://doi.org/10.1007/s00234-006-0183-z

3. Nagy Z, Nardai S (2017) Cerebral ischemia/repefusion injury: From bench space to bedside. Brain Res Bull 134:30-37. https://doi.org/10.1016/j.brainresbull.2017.06.011

4. Prabhakaran S, Ruff I, Bernstein RA (2015) Acute stroke intervention: a systematic review. JAMA 313(14):1451-1462. https://doi.org/10.1001/jama.2015.3058

5. Eltzschig HK, Eckle T (2011) Ischemia and reperfusion-from mechanism to translation. Nat Med 17(11):1391-1401. https://doi.org/10.1038/nm.2507

6. Broughton BR, Reutens DC, Sobey CG (2009) Apoptotic mechanisms after cerebral ischemia. Stroke 40(5):e331-e339. https://doi.org/10.1161/STROKEAHA.108.531632

7. Gupta R, Ghosh S (2017) Putative roles of mitochondrial Voltage-Dependent Anion Channel, Bcl-2 family proteins and c-Jun N-terminal Kinases in ischemic stroke associated apoptosis. Biochim Open 4:47-55. https://doi.org/10.1016/j.biopen.2017.02.002

8. D'Orsi B, Mateyka J, Prehn JHM (2017) Control of mitochondrial physiology and cell death by the Bcl-2 family proteins Bax and Bok. Neurochem Int 109:162-170. https://doi.org/10.1016/j.neuint.2017.03.010

9. Lu L, Zhang XG, Zhong LL, Chen ZX, Li Y, Zheng GQ, Bian ZX (2016) Acupuncture for neurogenesis in experimental ischemic stroke: a systematic review and meta-analysis. Sci Rep 6:19521. https://doi.org/10.1038/srep19521

10. Chang QY, Lin YW, Hsieh CL (2018) Acupuncture and neuroregeneration in ischemic stroke. Neural Regen Res 13(4):573-583. https://doi.org/10.4103/1673-5374.230272

11. Zhan J, Pan R, Zhou M, Tan F, Huang Z, Dong J, Wen Z (2018) Electroacupuncture as an adjunctive therapy for motor dysfunction in acute stroke survivors: a systematic review and meta-analyses. BMJ Open 8(1):e017153. https://doi.org/10.1136/bmjopen-2017-017153

12. Long M, Wang Z, Zheng D, Chen J, Tao W, Wang L, Yin N, Chen Z (2019) Electroacupuncture Pretreatment Elicits Neuroprotection Against Cerebral Ischemia-Reperfusion Injury in Rats Associated with Transient Receptor Potential Vanilloid 1-Mediated Anti-Oxidant Stress and Anti-Inflammation. Inflammation 42(5):1777-1787. https://doi.org/10.1007/s10753-019-01040-y

13. Zhang J, Liu K, Elmadhoun O, Ji X, Duan Y, Shi J, He X, Liu X, Wu D, Che R, Geng X, Ding Y (2018) Synergistically Induced Hypothermia and Enhanced Neuroprotection by Pharmacological and Physical Approaches in Stroke. Aging Dis 9(4):578-589. https://doi.org/10.14336/AD.2017.0817

14. Dai Z, Xiao J, Liu SY, Cui L, Hu GY, Jiang DJ (2008) Rutaecarpine inhibits hypoxia/reoxygenationinduced apoptosis in rat hippocampal neurons. Neuropharmacology 55(8):1307-1312. https://doi.org/10.1016/j.neuropharm.2008.08.030 
15. Akpınar H, Nazıroğlu M, Övey is, Çiğ B, Akpınar O (2016) The neuroprotective action of dexmedetomidine on apoptosis, calcium entry and oxidative stress in cerebral ischemia-induced rats: Contribution of TRPM2 and TRPV1 channels. Sci Rep 6:37196. https://doi.org/10.1038/srep37196

16. Pegorini S, Braida D, Verzoni C, Guerini-Rocco C, Consalez GG, Croci L, Sala M (2005) Capsaicin exhibits neuroprotective effects in a model of transient global cerebral ischemia in Mongolian gerbils. Br J Pharmacol 144(5):727-735. https://doi.org/10.1038/sj.bjp.0706115

17. Shen MH, Zhang CB, Zhang JH, Li PF (2016) Electroacupuncture Attenuates Cerebral Ischemia and Reperfusion Injury in Middle Cerebral Artery Occlusion of Rat via Modulation of Apoptosis, Inflammation, Oxidative Stress, and Excitotoxicity. Evid Based Complement Alternat Med 2016:9438650. https://doi.org/10.1155/2016/9438650

18. Gidday JM (2006) Cerebral preconditioning and ischaemic tolerance. Nat Rev Neurosci 7(6):437448. https://doi.org/10.1038/nrn1927

19. Wang Q, Liang D, Wang F, Li W, Han Y, Zhang W, Xie Y, Xin W, Zhou B, Sun D, Cao F, Xiong L (2015) Efficacy of electroacupuncture pretreatment for myocardial injury in patients undergoing percutaneous coronary intervention: A randomized clinical trial with a 2-year follow-up. Int J Cardiol 194:28-35. https://doi.org/10.1016/j.ijcard.2015.05.043

20. Li MK, Li YJ, Zhang GF, Chen JQ, Zhang JP, Qi J, Huang Y, Lai XS, Tang CZ (2015) Acupuncture for ischemic stroke: cerebellar activation may be a central mechanism following Deqi. Neural Regen Res 10(12):1997-2003. https://doi.org/10.4103/1673-5374.172318

21. Xiong L, Lu Z, Hou L, Zheng H, Zhu Z, Wang Q, Chen S (2003) Pretreatment with repeated electroacupuncture attenuates transient focal cerebral ischemic injury in rats. Chin Med $\mathrm{J}$ (Engl) 116(1):108-111

22. Wang WW, Xie CL, Lu L, Zheng GQ (2014) A systematic review and meta-analysis of Baihui (GV20)based scalp acupuncture in experimental ischemic stroke. Sci Rep 4:3981. https://doi.org/10.1038/srep03981

23. Mao C, Hu C, Zhou Y, Zou R, Li S, Cui Y, Tian W (2020) Electroacupuncture Pretreatment against Cerebral Ischemia/Reperfusion Injury through Mitophagy. Evid Based Complement Alternat Med 2020:7486041. https://doi.org/10.1155/2020/7486041

24. Zhang BY, Wang GR, Ning WH, Liu J, Yang S, Shen Y, Wang Y, Zhao MX, Li L (2020) Electroacupuncture Pretreatment Elicits Tolerance to Cerebral Ischemia/Reperfusion through Inhibition of the GluN2B/m-Calpain/p38 MAPK Proapoptotic Pathway. Neural Plast 2020:8840675. https://doi.org/10.1155/2020/8840675

25. Jung YS, Lee SW, Park JH, Seo HB, Choi BT, Shin HK (2016) Electroacupuncture preconditioning reduces ROS generation with NOX4 down-regulation and ameliorates blood-brain barrier disruption after ischemic stroke. J Biomed Sci 23:32. https://doi.org/10.1186/s12929-016-0249-0

26. Mei ZG, Huang YG, Feng ZT, Luo YN, Yang SB, Du LP, Jiang K, Liu XL, Fu XY, Deng YH, Zhou HJ (2020) Electroacupuncture ameliorates cerebral ischemia/reperfusion injury by suppressing 
autophagy via the SIRT1-FOXO1 signaling pathway. Aging 12(13):13187-13205.

https://doi.org/10.18632/aging.103420

27. Li X, Luo P, Wang Q, Xiong L (2012) Electroacupuncture Pretreatment as a Novel Avenue to Protect Brain against Ischemia and Reperfusion Injury. Evid Based Complement Alternat Med 2012:195397. https://doi.org/10.1155/2012/195397

28. Xing Y, Zhang M, Li WB, Dong F, Zhang F (2018) Mechanisms Involved in the Neuroprotection of Electroacupuncture Therapy for Ischemic Stroke. Front Neurosci 12:929.

https://doi.org/10.3389/fnins.2018.00929

29. Chen C, Yu Q, Xu K, Cai L, Felicia BM, Wang L, Zhang A, Dai Q, Geng W, Wang J, Mo Y (2020) Electroacupuncture pretreatment prevents ischemic stroke and inhibits Wnt signaling-mediated autophagy through the regulation of GSK-3 $\beta$ phosphorylation. Brain Res Bull 158:90-98. https://doi.org/10.1016/j.brainresbull.2020.03.002

30. Cai W, Shen WD (2018) Anti-Apoptotic Mechanisms of Acupuncture in Neurological Diseases: A Review. Am J Chin Med 46(3):515-535. https://doi.org/10.1142/S0192415X1850026X

31. Uzdensky AB (2019) Apoptosis regulation in the penumbra after ischemic stroke: expression of proand antiapoptotic proteins. Apoptosis 24(9-10):687-702. https://doi.org/10.1007/s10495-01901556-6

32. Chavez LM, Huang SS, MacDonald I, Lin JG, Lee YC, Chen YH (2017) Mechanisms of Acupuncture Therapy in Ischemic Stroke Rehabilitation: A Literature Review of Basic Studies. Int J Mol Sci 18(11):2270. https://doi.org/10.3390/ijms18112270

33. Wang MM, Zhang M, Feng YS, Xing Y, Tan ZX, Li WB, Dong F, Zhang F (2020) Electroacupuncture Inhibits Neuronal Autophagy and Apoptosis via the PI3K/AKT Pathway Following Ischemic Stroke. Front Cell Neurosci 14:134. https://doi.org/10.3389/fncel.2020.00134

34. Zhao Y, Deng B, Li Y, Zhou L, Yang L, Gou X, Wang Q, Chen G, Xu H, Xu L (2015) Electroacupuncture Pretreatment Attenuates Cerebral Ischemic Injury via Notch Pathway-Mediated Up-Regulation of Hypoxia Inducible Factor-1a in Rats. Cell Mol Neurobiol 35(8):1093-1103. https://doi.org/10.1007/s10571-015-0203-9

35. Han P, Korepanova AV, Vos MH, Moreland RB, Chiu ML, Faltynek CR (2013) Quantification of TRPV1 protein levels in rat tissues to understand its physiological roles. J Mol Neurosci 50(1):23-32. https://doi.org/10.1007/s12031-012-9849-7

36. Randhawa PK, Jaggi AS (2018) A Review on Potential Involvement of TRPV ${ }_{1}$ Channels in IschemiaReperfusion Injury. J Cardiovasc Pharmacol Ther 23(1):38-45.

https://doi.org/10.1177/1074248417707050

37. Miyanohara J, Shirakawa H, Sanpei K, Nakagawa T, Kaneko S (2015) A pathophysiological role of TRPV1 in ischemic injury after transient focal cerebral ischemia in mice. Biochem Biophys Res Commun 467(3):478-483. https://doi.org/10.1016/j.bbrc.2015.10.027

38. Hakimizadeh E, Shamsizadeh A, Roohbakhsh A, Arababadi MK, Hajizadeh MR, Shariati M, Rahmani MR, Allahtavakoli M (2017) Inhibition of transient receptor potential vanilloid-1 confers 
neuroprotection, reduces tumor necrosis factor-alpha, and increases IL-10 in a rat stroke model. Fundam Clin Pharmacol 31(4):420-428. https://doi.org/10.1111/fcp.12279

39. Hakimizadeh E, Shamsizadeh A, Roohbakhsh A, Arababadi MK, Hajizadeh MR, Shariati M, Fatemi I, Moghadam-Ahmadi A, Bazmandegan G, Rezazadeh H, Allahtavakoli M (2017) TRPV1 receptormediated expression of Toll-like receptors 2 and 4 following permanent middle cerebral artery occlusion in rats. Iran J Basic Med Sci 20(8):863-869. https://doi.org/10.22038/IJBMS.2017.9107

40. Lu KW, Hsu CK, Hsieh CL, Yang J, Lin YW (2016) Probing the Effects and Mechanisms of Electroacupuncture at Ipsilateral or Contralateral ST36-ST37 Acupoints on CFA-induced Inflammatory Pain. Sci Rep 6:22123. https://doi.org/10.1038/srep22123

41. Inprasit C, Lin YW (2020) TRPV1 Responses in the Cerebellum Lobules V, Vla and VII Using Electroacupuncture Treatment for Inflammatory Hyperalgesia in Murine Model. Int J Mol Sci 21(9):3312. https://doi.org/10.3390/ijms21093312

42. Yang J, Hsieh CL, Lin YW (2017) Role of Transient Receptor Potential Vanilloid 1 in Electroacupuncture Analgesia on Chronic Inflammatory Pain in Mice. Biomed Res Int 2017:5068347. https://doi.org/10.1155/2017/5068347

43. Du J, Fang J, Xiang X, Yu J, Le X, Liang Y, Jin X, Fang J (2020) Effects of low- and high-frequency electroacupuncture on protein expression and distribution of TRPV1 and P2X3 in rats with peripheral nerve injury. Acupunct Med 964528420968845. https://doi.org/10.1177/0964528420968845

44. Ji B, Hu J, Ma S (2013) Effects of electroacupuncture Zusanli (ST36) on food intake and expression of POMC and TRPV1 through afferents-medulla pathway in obese prone rats. Peptides 40:188-194. https://doi.org/10.1016/j.peptides.2012.10.009

45. Lin YW, Hsieh CL (2010) Electroacupuncture at Baihui acupoint (GV20) reverses behavior deficit and long-term potentiation through $\mathrm{N}$-methyl-d-aspartate and transient receptor potential vanilloid subtype 1 receptors in middle cerebral artery occlusion rats. J Integr Neurosci 9(3):269-282. https://doi.org/10.1142/s0219635210002433

\section{Figures}


a

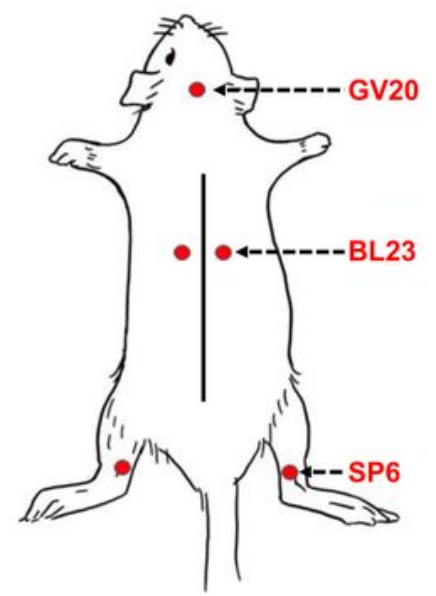

b

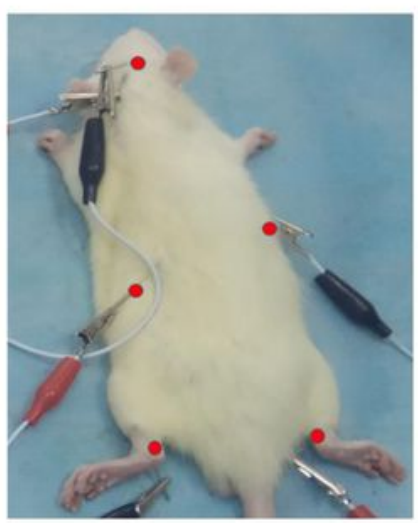

C

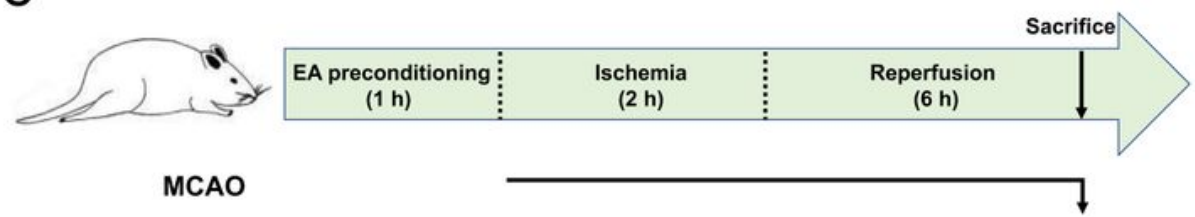

EA+MCAO

d

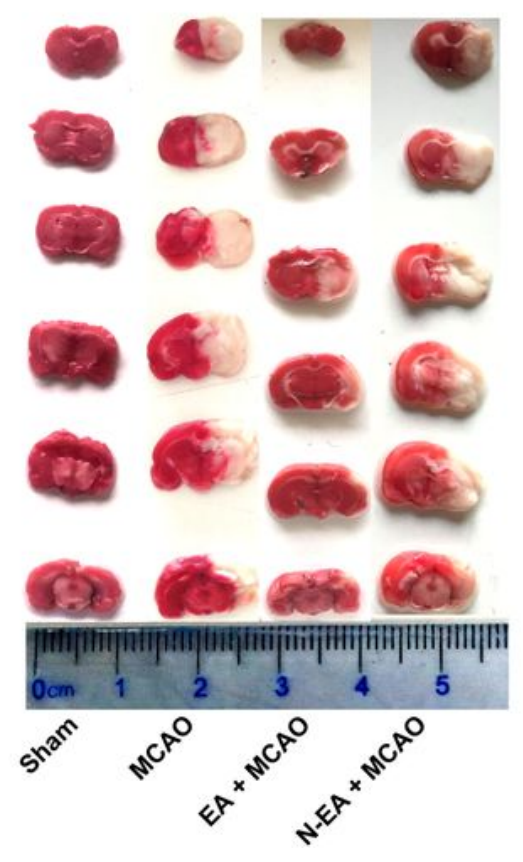

e

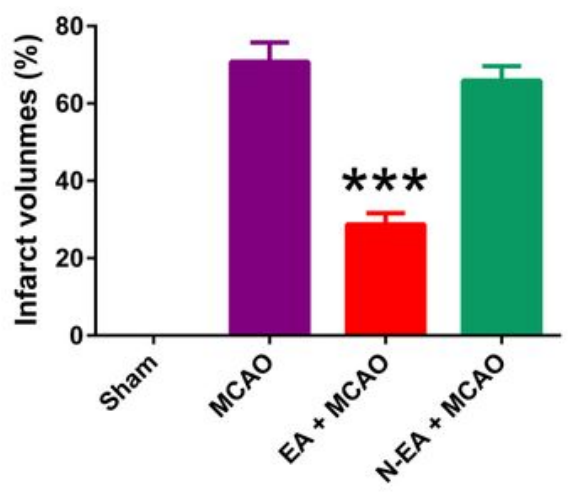

\section{Figure 1}

EA pretreatment attenuates cerebral ischemia-reperfusion injury in MCAO rats. (a) Location of GV20, BL23 and SP6 acupoints in rat. (b) EA stimulation in SD rats. Red circles indicated the corresponding acupoints as mentioned above. (c) Schematic diagram of the experiment I as described in experimental protocols. (d) Representative TTC staining showed noninfarct (red) and infarct (white) regions of brain tissue section in the different groups at $6 \mathrm{~h}$ after reperfusion. (e) Quantification of infarct volume among 
the four groups was statistically analyzed ( ${ }^{\star \star *} \mathrm{P}<0.001$ vs. MCAO; $n=8 /$ group, data were expressed as mean $\pm S D$ ).

\section{a}
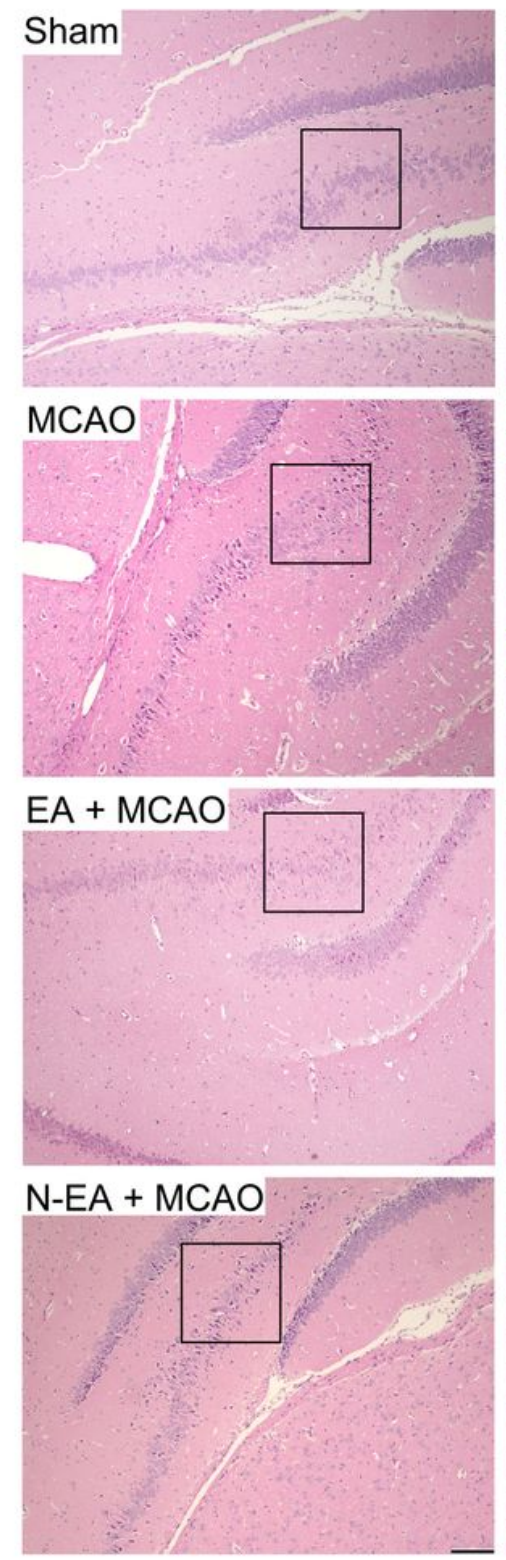
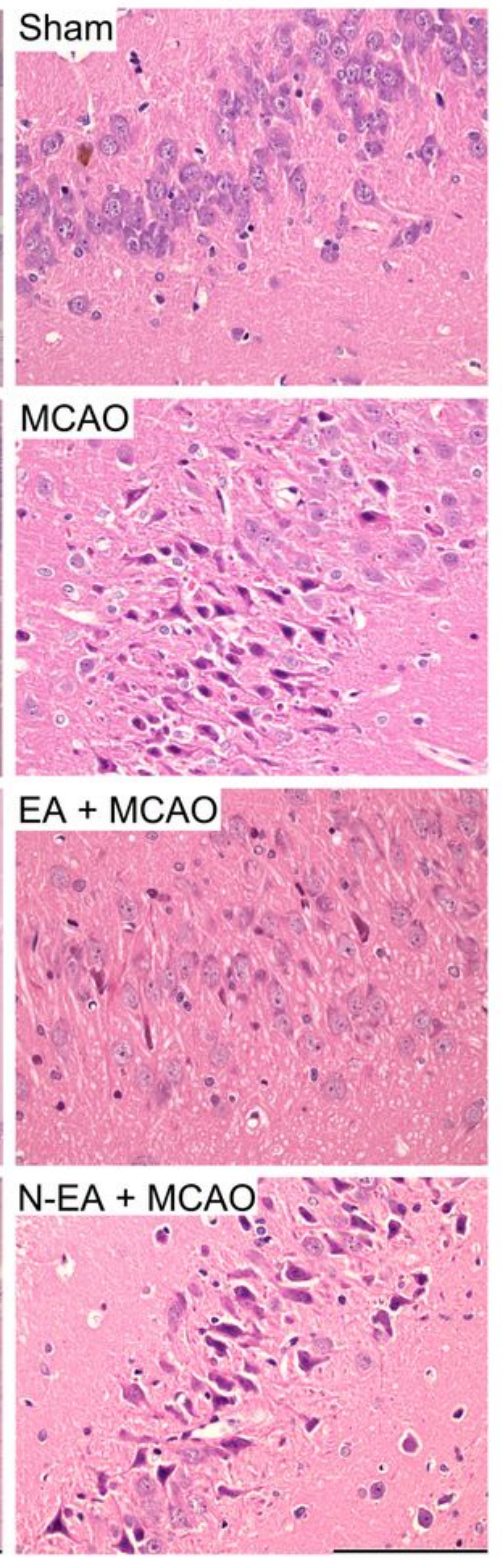

b
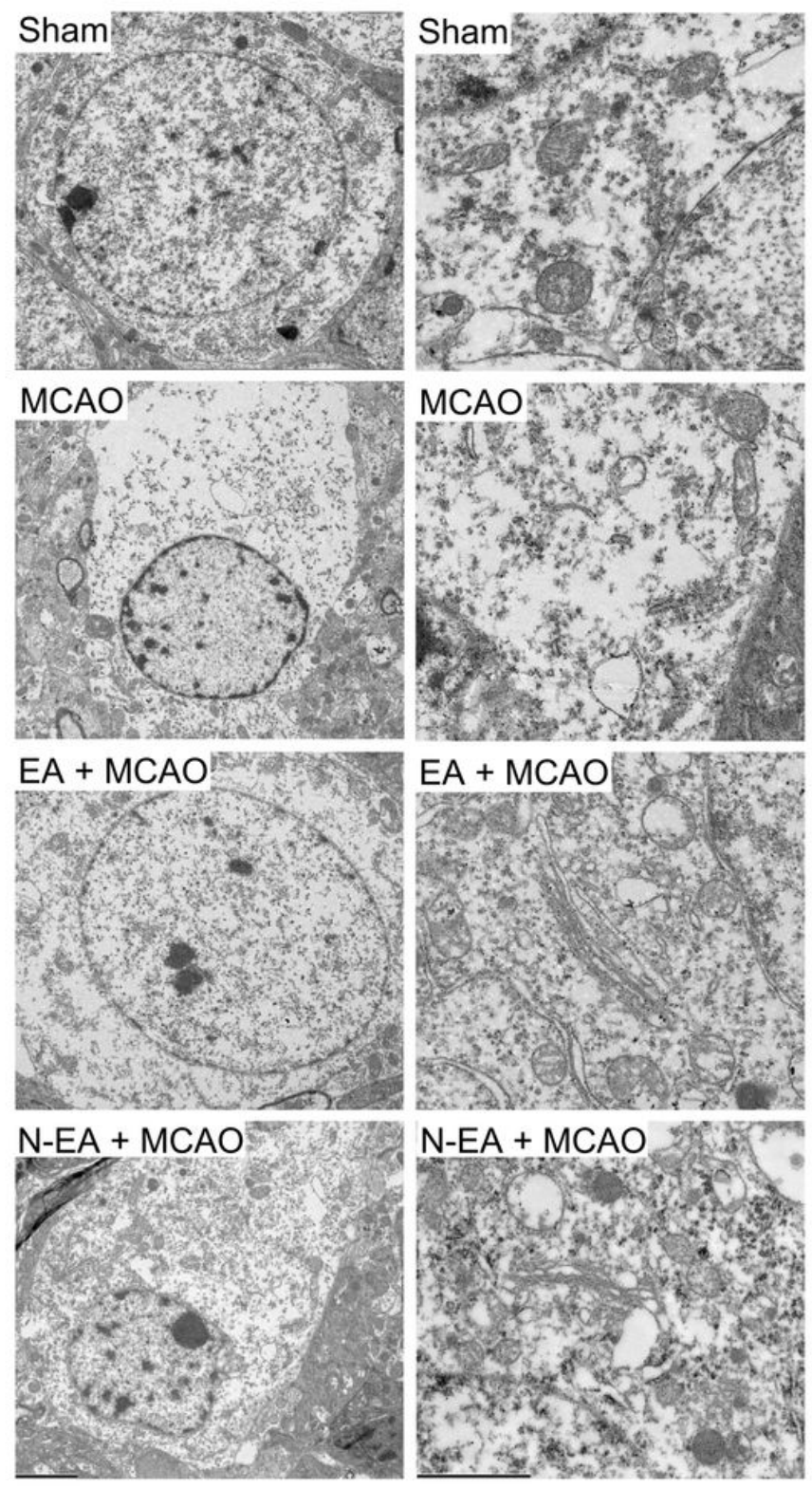

\section{Figure 2}

EA pretreatment evokes neuroprotection against cerebral ischemia-reperfusion injury. (a) Representative HE staining showed nerve cell injury in rat hippocampus (Left, $\times 100$ and Right, $\times 400$ ), such as nuclear pyknosis (green arrows) and reactive hyperplasia of glial cells (yellow arrows). Scale bar $=50 \mu \mathrm{m}$. (b) Neurons (left panel, scale bar $=2 \mu \mathrm{m}$ ) and mitochondrial ultrastructure of neurons (right panel, scale bar $=1 \mu \mathrm{m}$ ) in the hippocampus were observed under transmission electron microscope. (Left, $\times 1700$ and Right, ×5000). 
a

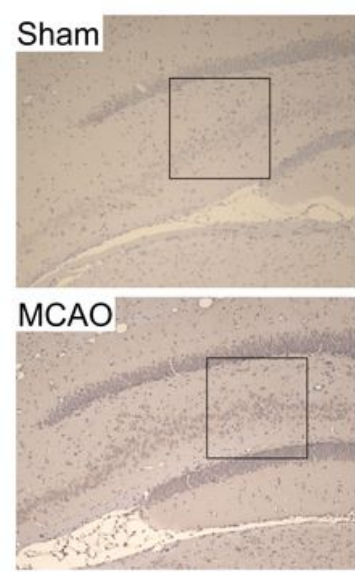

$E A+M C A O$

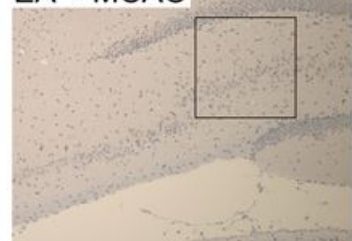

$\mathrm{N}-\mathrm{EA}+\mathrm{MCAO}$

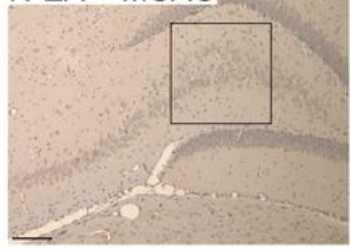

Bax
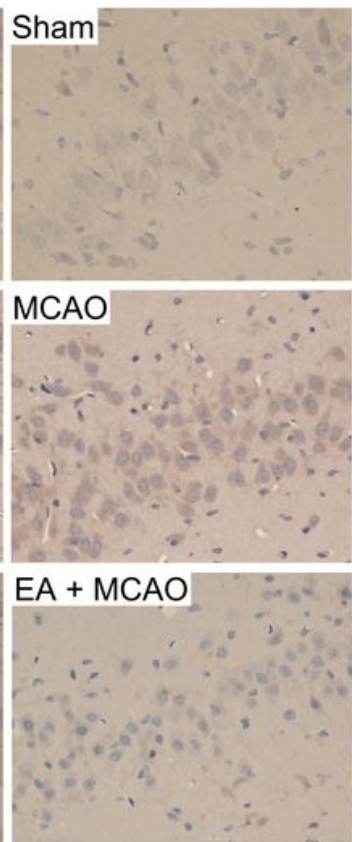

$\mathrm{N}-\mathrm{EA}+\mathrm{MCAO}$
Bcl-2
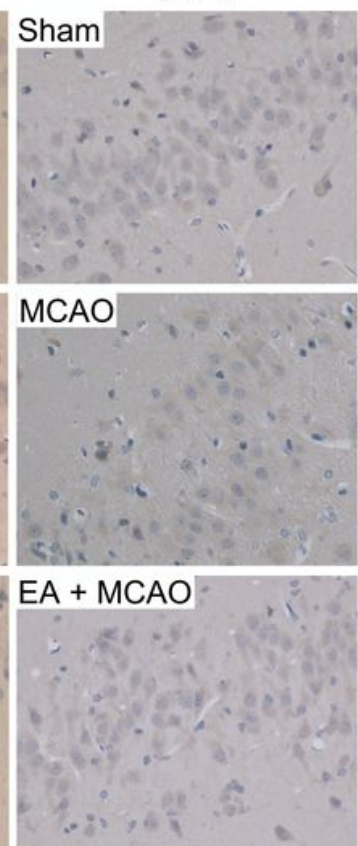

$\mathrm{N}-\mathrm{EA}+\mathrm{MCAO}$
Cleaved caspase-3

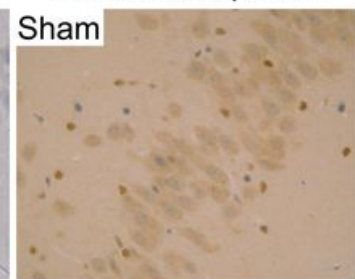

MCAO

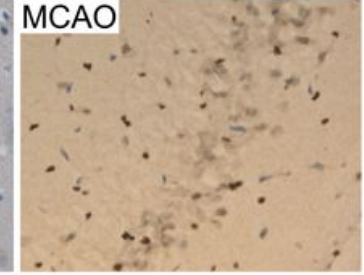

$E A+M C A O$

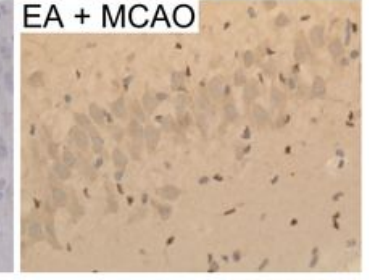

$\mathrm{N}-\mathrm{EA}+\mathrm{MCAO}$ b

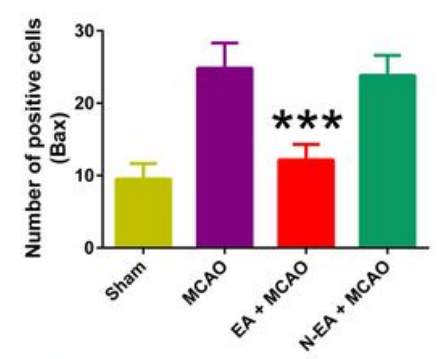

C

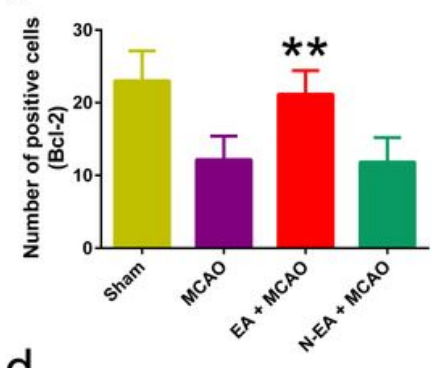

d

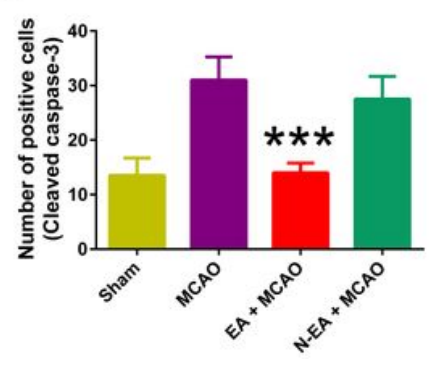

Figure 3

EA pretreatment inhibits hippocampal neuronal apoptosis in MCAO rats. (a) Representative immunohistochemical staining showed apoptosis-related protein expression in rat hippocampus, such as Bax, Bcl-2, and cleaved caspase-3. The apoptosis-positive cells were stained in brown or brown-yellow. Scale bar $=50 \mu \mathrm{m}$. (b-d) Statistical analysis of Bax-, Bcl-2-, and cleaved caspase-3-positive cells in the different groups was shown, separately. ( ${ }^{*} P<0.01$ and ${ }^{\star * *} P<0.001$ vs. MCAO; $n=5 /$ group, data were expressed as mean $\pm S D$ ). 
a

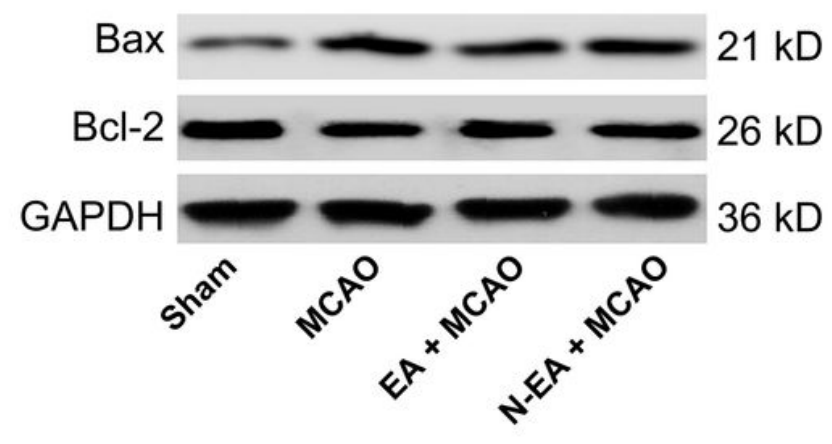

C

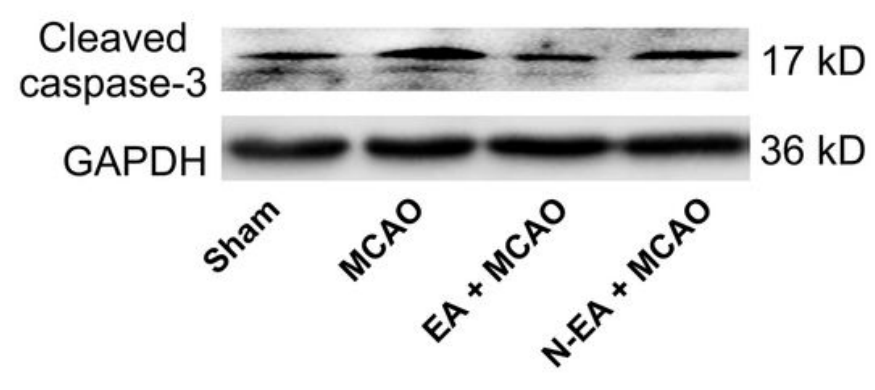

b

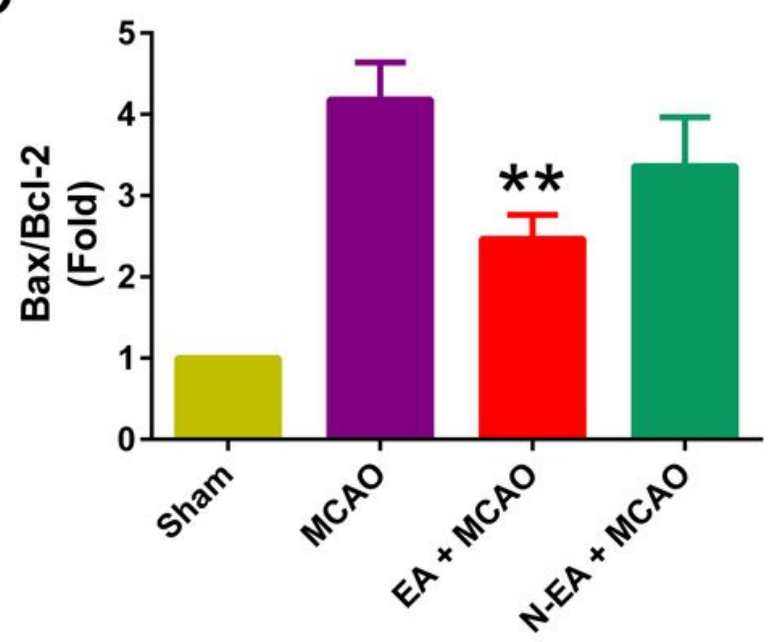

d

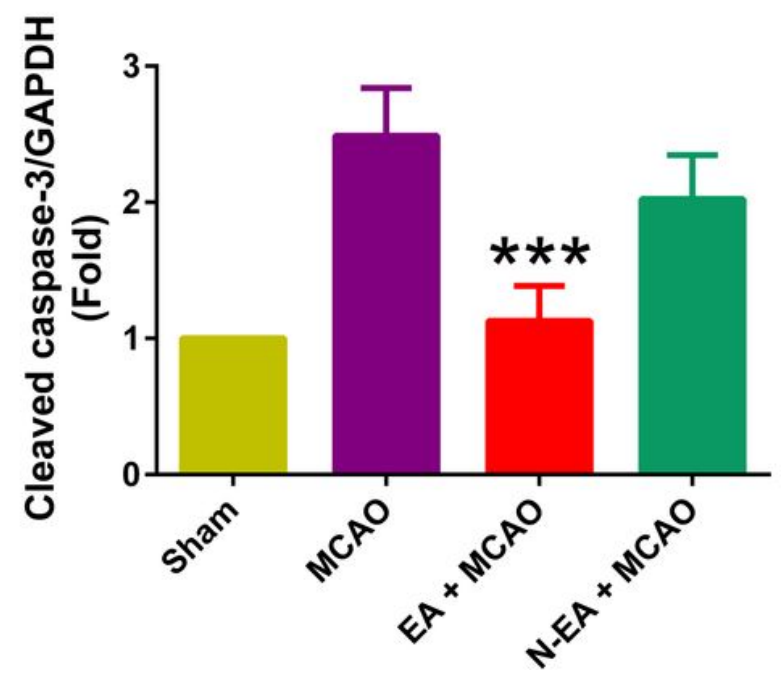

Figure 4

EA pretreatment inhibits apoptosis-related protein expression of hippocampal neuronal in MCAO rats. (a, c) Representative Western blot showed the expression of Bax, Bcl-2, and cleaved caspase- 3 proteins in rat hippocampus. (b, d) Relative protein expression of Bax/Bcl-2 and cleaved caspase-3/GAPDH was statistically analyzed, respectively ( ${ }^{\star \star} P<0.01$ and ${ }^{\star \star *} P<0.001$ vs. MCAO; $n=5 /$ group, data were expressed as mean $\pm \mathrm{SD}$ ). 


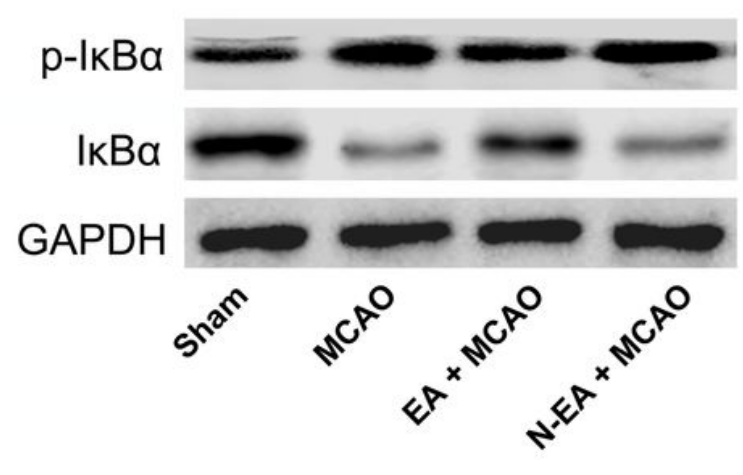

$40 \mathrm{kD}$

$39 \mathrm{kD}$

$36 \mathrm{kD}$

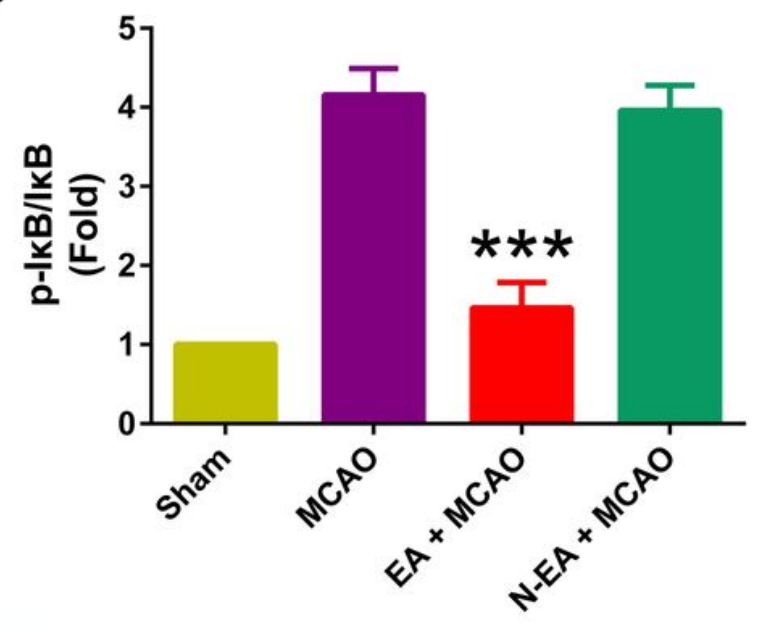

C

p-NF-kB p65

$65 \mathrm{kD}$

Histone $\mathrm{H} 3$

$15 \mathrm{kD}$

NF-KB p65

$65 \mathrm{kD}$

GAPDH

$36 \mathrm{kD}$
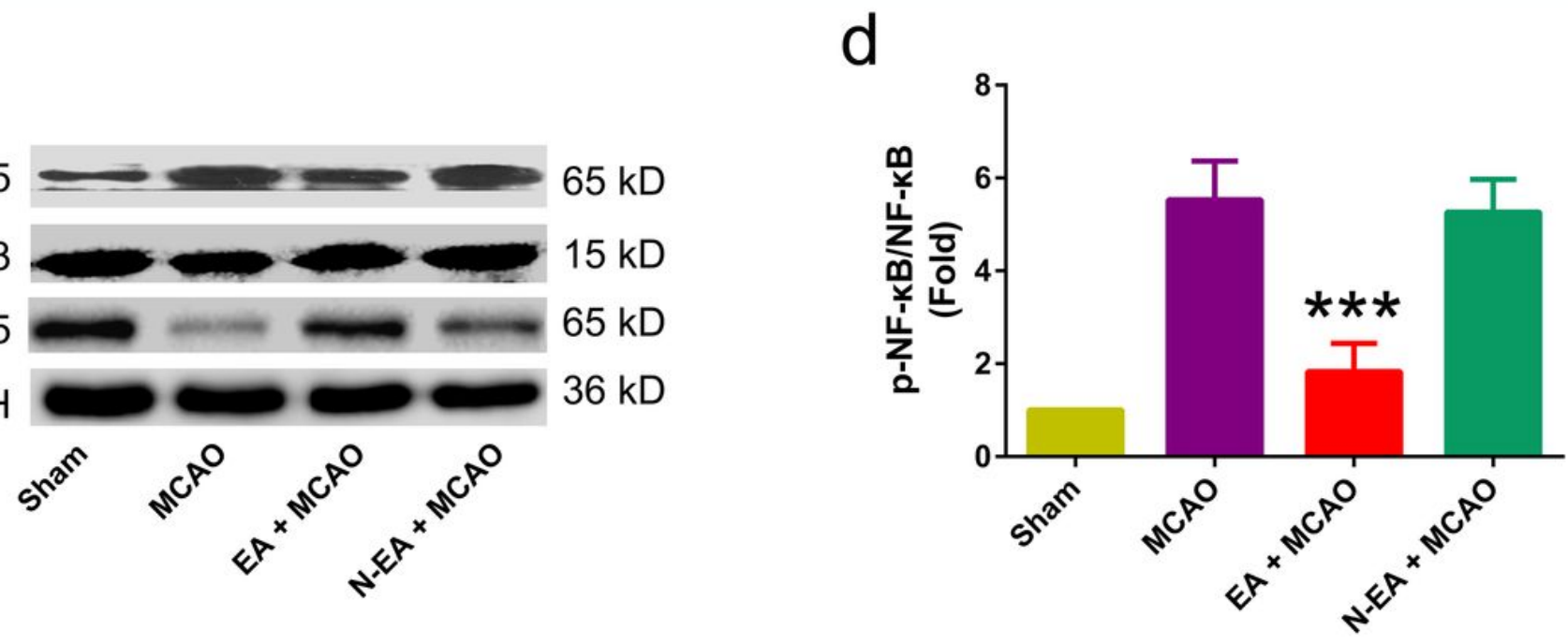

\section{Figure 5}

EA pretreatment curbs NF-KB signaling activation in MCAO rats. (a) Cytoplasmic protein expression of $\mathrm{I \kappa Ba}$ and $\mathrm{p}-\mathrm{I} \mathrm{KBa}$ were detected by Western blot. (b) Relative protein expression of $\mathrm{p}-\mathrm{I} \mathrm{KBa} / \mathrm{I} \mathrm{Ka}$ was statistically analyzed. (c) Nuclear protein expression of p-NF-KB (p65) and cytoplasmic protein expression of NF-kB (p65) were detected by Western blot. (d) Relative protein expression of p-NF-kB (p65)/NF-kB (p65) was statistically analyzed ( ${ }^{* \star *} P<0.001$ vs. MCAO; $n=5 /$ group, data were expressed as mean \pm $\mathrm{SD})$. 
a

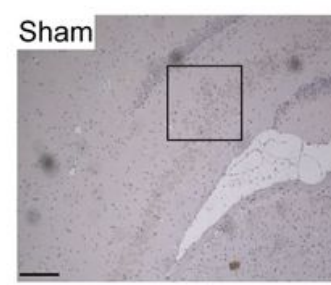

Sham

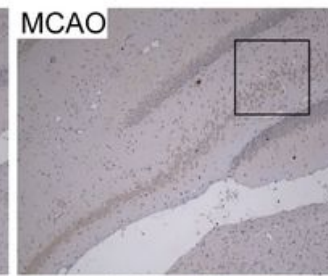

$\mathrm{MCAO}$

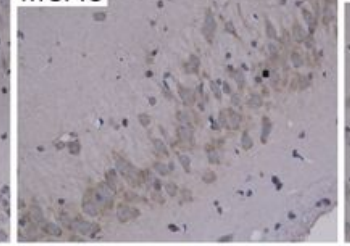

$\mathrm{EA}+\mathrm{MCAO}$

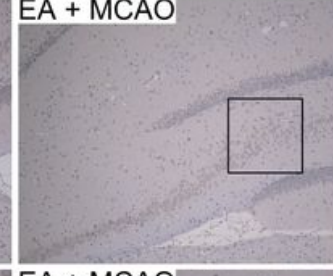

$E A+M C A O$

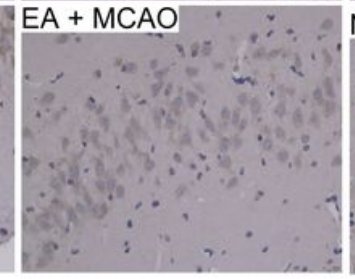

$\mathrm{N}-\mathrm{EA}+\mathrm{MCAO}$

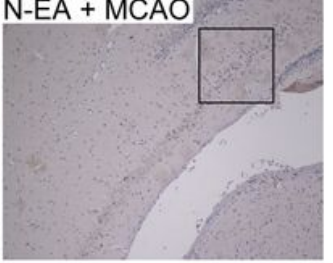

$\mathrm{N}-\mathrm{EA}+\mathrm{MCAO}$

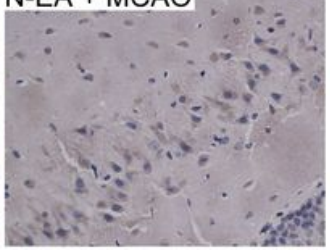

C

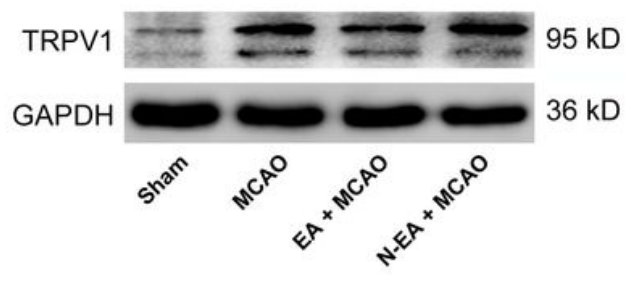

b
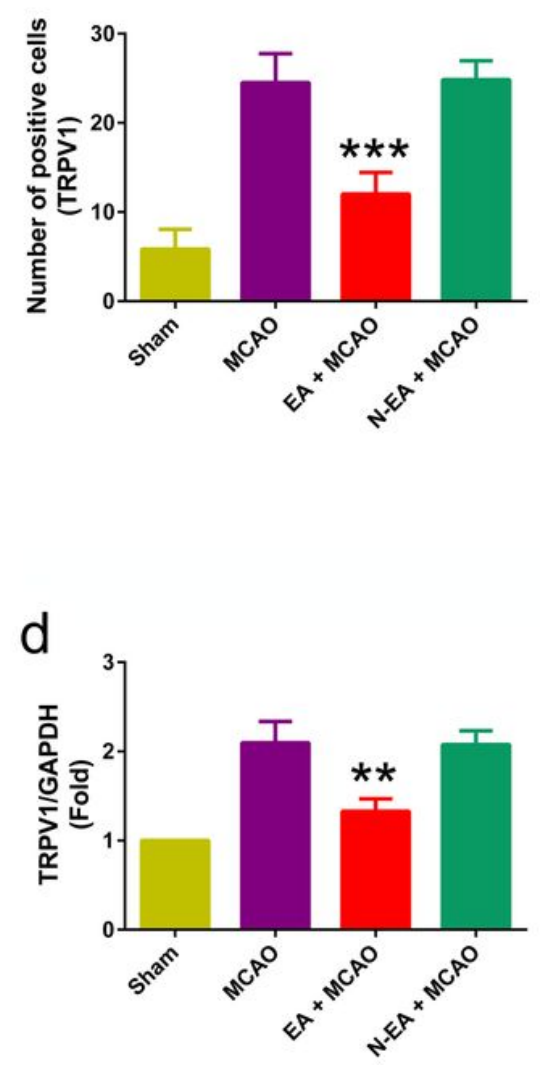

Figure 6

EA pretreatment reduces TRPV1 expression in the hippocampus of MCAO rats. (a) Representative immunohistochemical staining showed TRPV1 expression in rat hippocampus (Up, $\times 100$ and Down, $\times 400$ ). TRPV1-positive cells were stained in brown or brown-yellow. Scale bar $=50 \mu \mathrm{m}$. (b) Statistical analysis of TRPV1-positive cells in the different groups was shown. (c) The protein expression of TRPV-1 was assessed by Western blot and (d) statistical analysis was shown ( ${ }^{\star \star P} \mathrm{P}<0.01$ and ${ }^{* \star *} \mathrm{P}<0.001$ vs. MCAO; $n=5$ /group, data were expressed as mean $\pm S D$ ). 


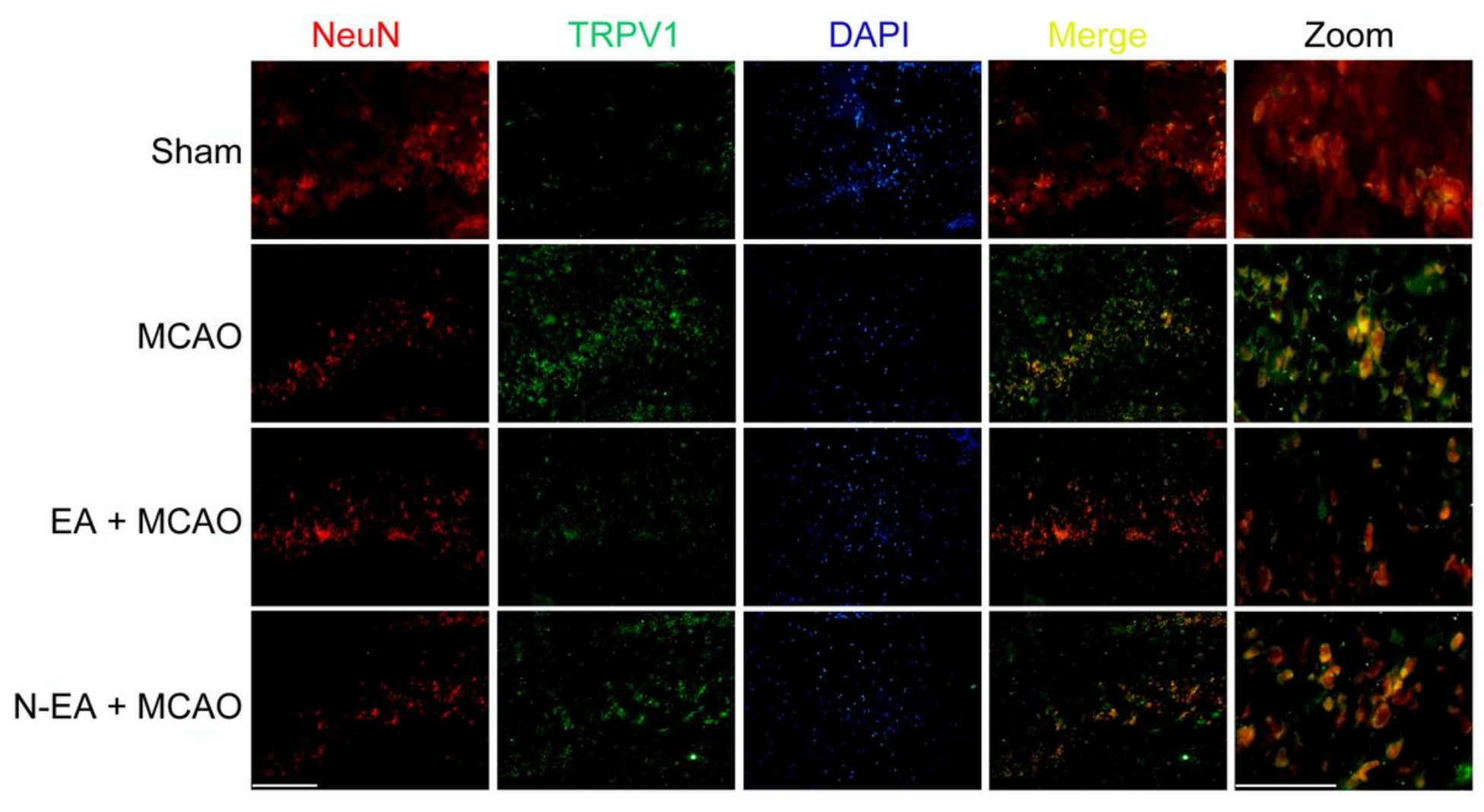

Figure 7

Colocalization of TRPV1 and NeuN in the hippocampal neurons. Immunofluorescence images of brain tissue sections showed colocalization of TRPV1 (green) and NeuN (red) in the hippocampal neurons. Cell nuclei were stained with DAPI (blue). Merge panels were overlay images presenting triple-labeled cells (yellow). Zoom panels were magnified images of the merge panels. Scar bar $=100 \mu \mathrm{m}$.

a

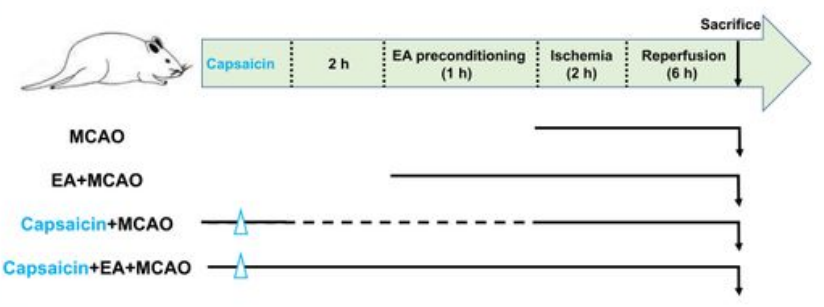

d

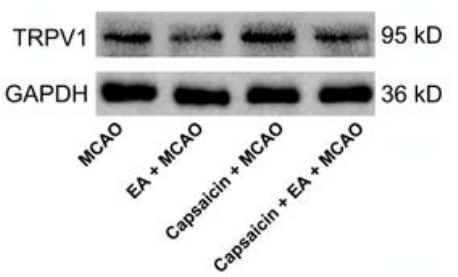

e

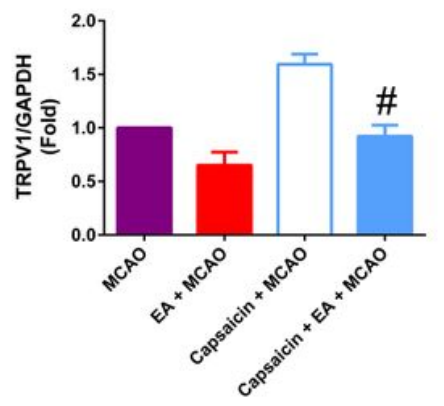

b

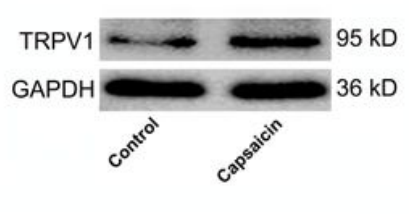

C

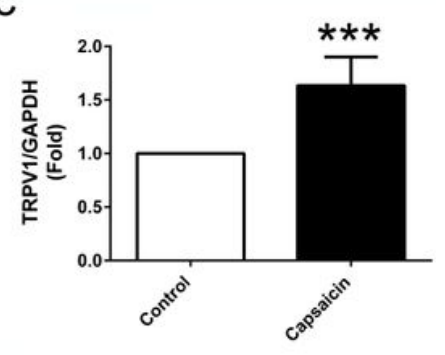

g

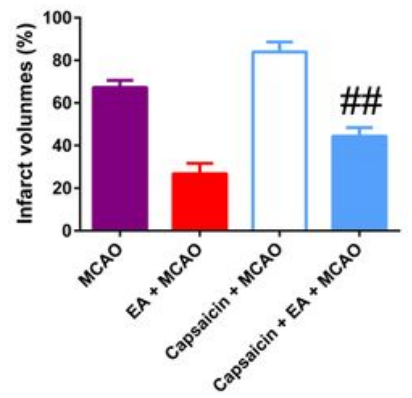




\section{Figure 8}

Capsaicin abrogates EA pretreatment-evoked neuroprotection against cerebral ischemia-reperfusion injury in MCAO rats. (a) Schematic diagram of the experiment II as described in experimental protocols. (b, c) Representative Western blot showed TRPV1 expression in rats with or without capsaicin (TRPV1 agonists) application and statistical analysis was shown. (d) TRPV1 expression in the different groups were detected by Western blot. (e) Relative protein expression of TRPV1/GAPDH was statistically analyzed. (f) Representative TTC staining showed noninfarct (red) and infarct (white) regions of brain tissue section in the different groups. (g) Quantitative analysis of infarct volume in the four groups of rats was shown ( ${ }^{\star \star \star} \mathrm{P}<0.001$ vs. Control, \#P $<0.05$ and \#\#P $<0.01$ vs. $E A+M C A O ; n=8 /$ group, data were expressed as mean $\pm \mathrm{SD})$. 
a

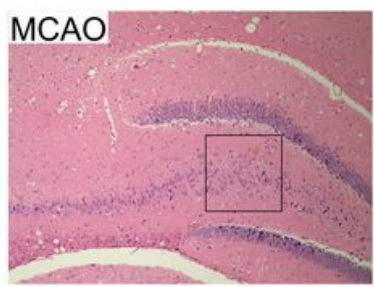

$\mathrm{EA}+\mathrm{MCAO}$

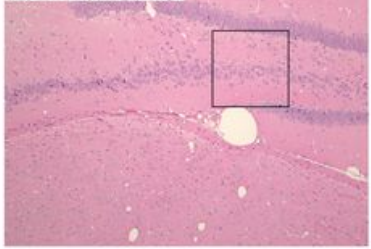

Capsaicin + MCAO

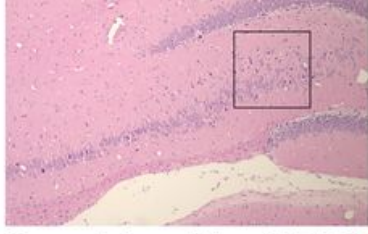

Capsaicin + EA + MCAO

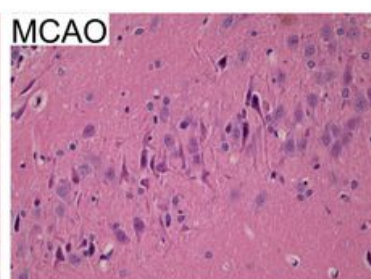

$\mathrm{EA}+\mathrm{MCAO}$

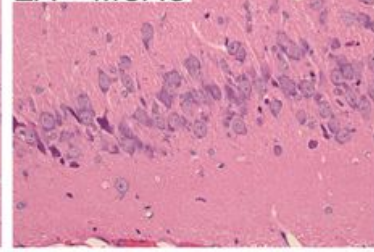

Capsaicin + MCAO

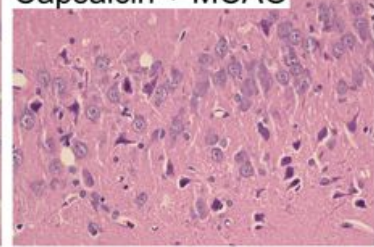

Capsaicin + EA + MCAO

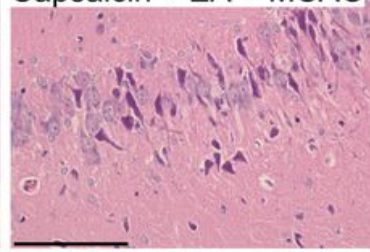

b
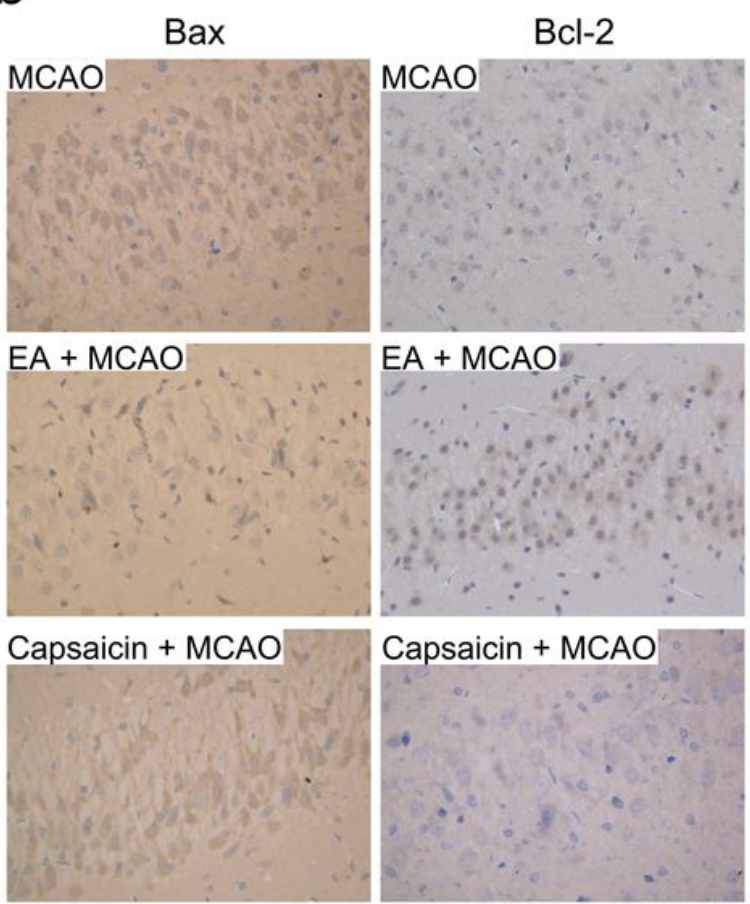

Capsaicin + EA + MCAO

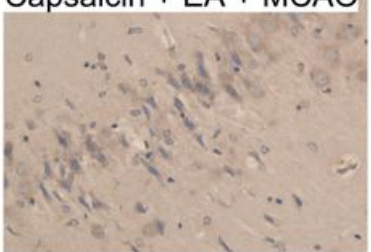

Cleaved caspase-3

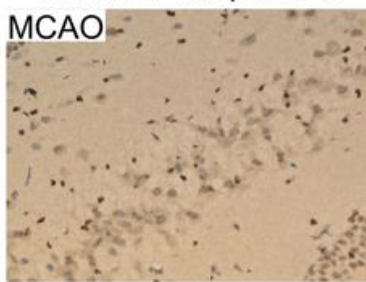

$\mathrm{EA}+\mathrm{MCAO}$

$E A+M C A O$

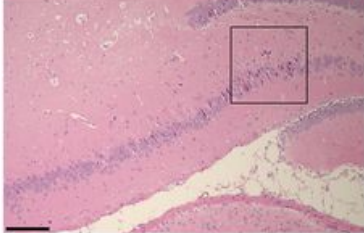

c

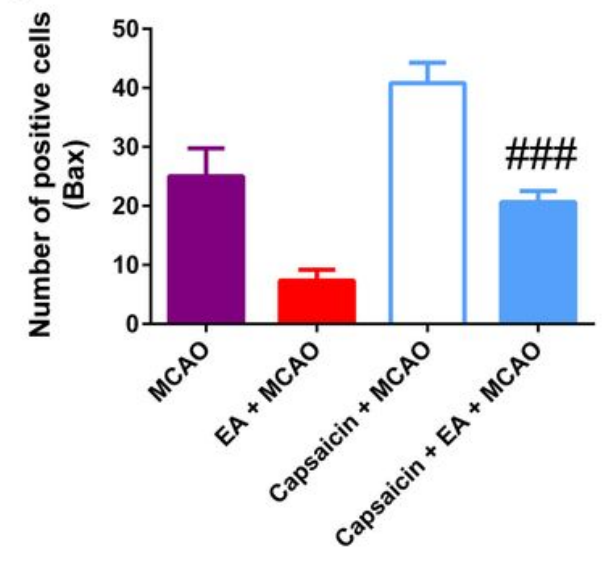

d

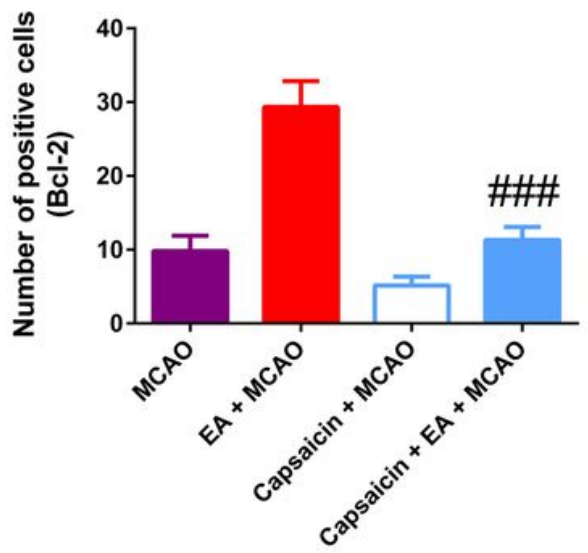

e

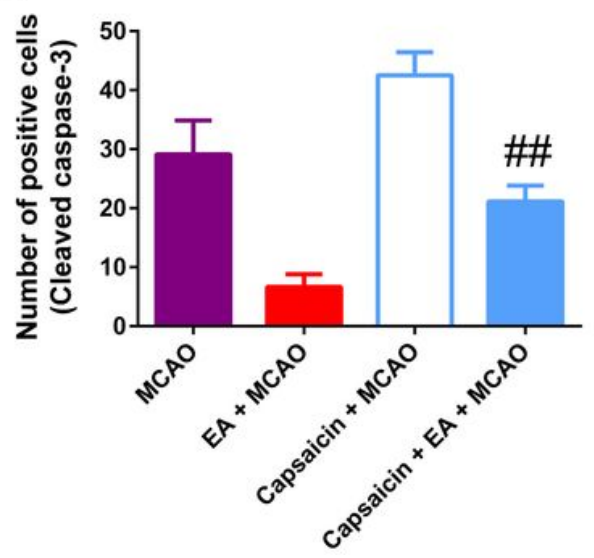

Figure 9

Capsaicin abolishes anti-apoptosis activity of EA pretreatment in MCAO rats. (a) Representative HE staining showed nerve cell injury in hippocampus of MCAO rats with EA pretreatment after capsaicin application (Left, $\times 100$ and Middle, $\times 400$ ). Green arrows indicated neuron injury and yellow arrows represented glial cell hyperplasia. (b) Representative immunohistochemical staining showed apoptosisrelated protein expression in rat hippocampus, such as Bax, Bcl-2, and cleaved caspase-3. The positive cells were stained in brown or brown-yellow. Scale bar $=50 \mu \mathrm{m}$. (c-e) Statistical analysis of Bax-, Bcl-2-, 
and cleaved caspase-3-positive cells in the different groups was shown, separately (\#\#P $<0.01$ and \#\#\#P $<0.001$ vs. EA + MCAO; $n=5$ /group, data were expressed as mean $\pm S D$ ).

a

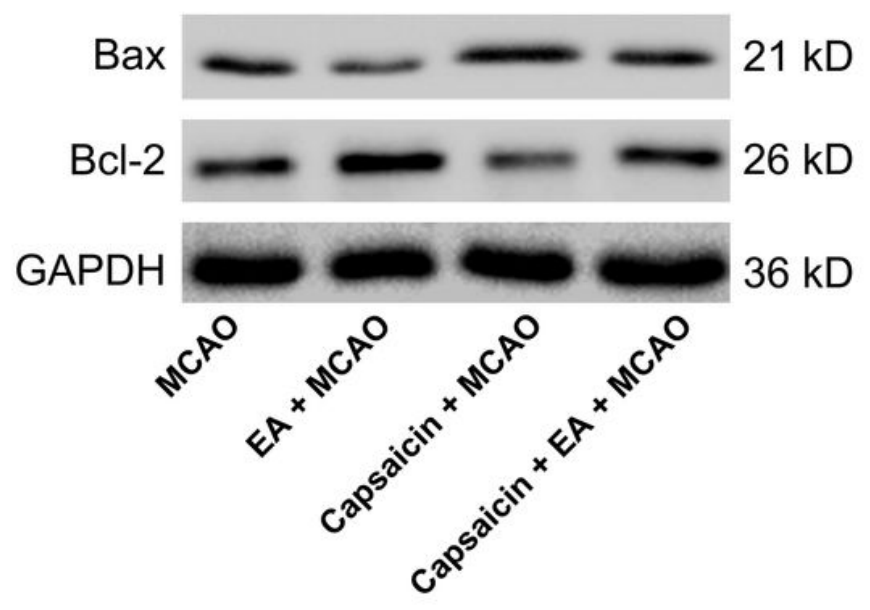

C

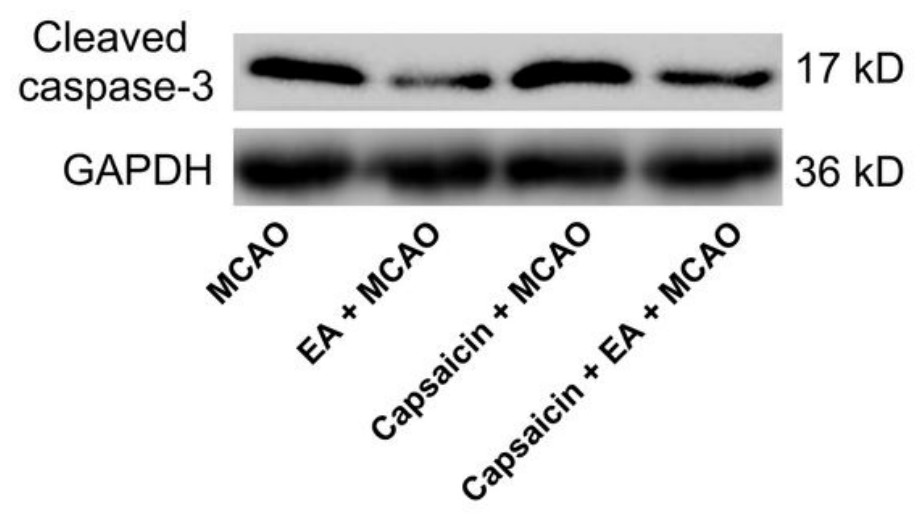

b

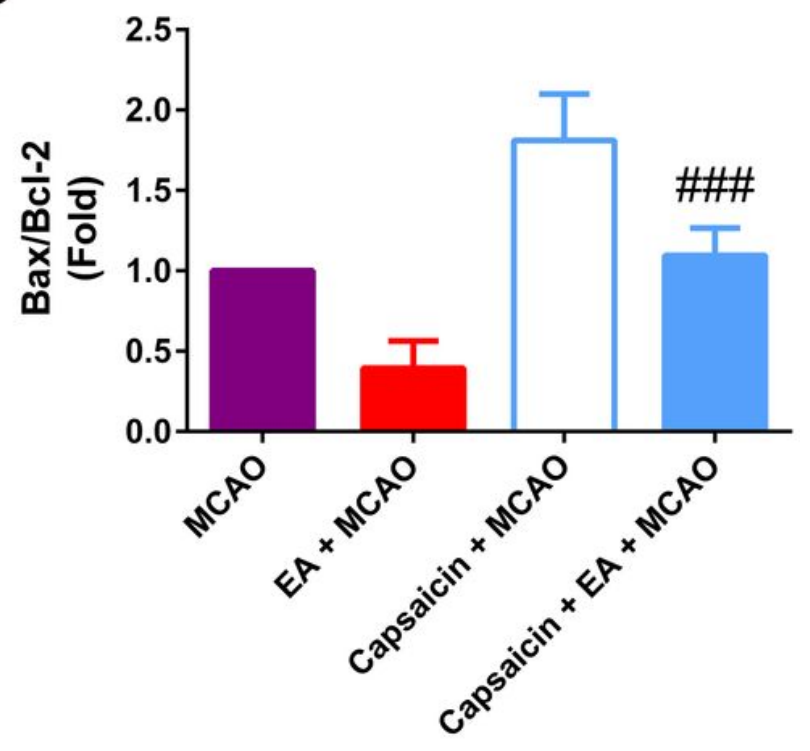

d

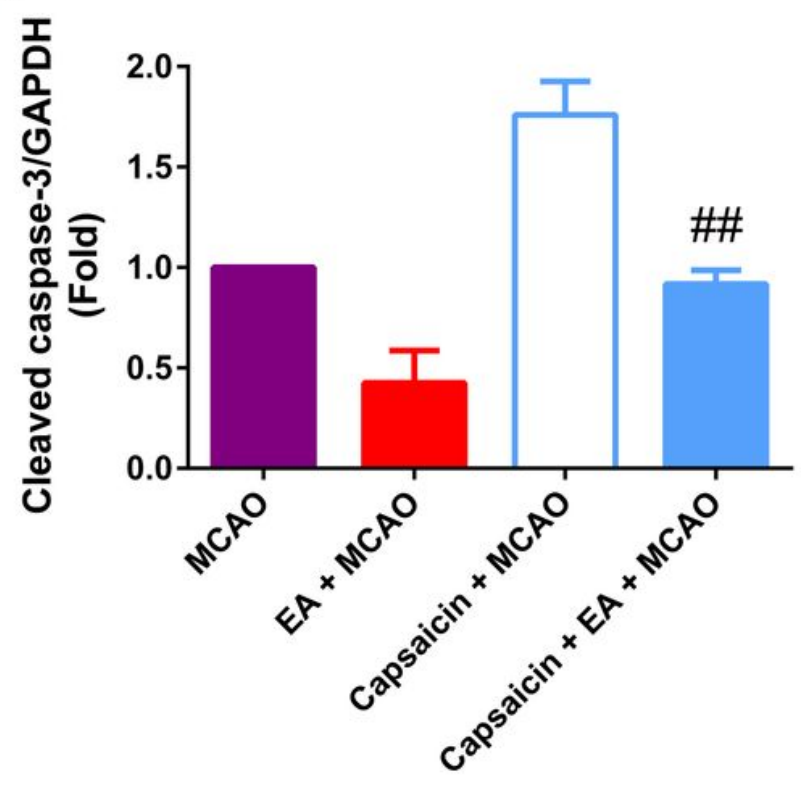

Figure 10

Capsaicin reverses the inhibitive effect of EA pretreatment on apoptosis-related protein expression in MCAO rats. $(\mathrm{a}, \mathrm{c})$ Representative Western blot showed the expression of $\mathrm{Bax}, \mathrm{Bcl}-2$, and cleaved caspase3 proteins in rat hippocampus. $(b, d)$ Relative protein expression of $\mathrm{Bax} / \mathrm{Bcl}-2$ and cleaved caspase3/GAPDH was statistically analyzed, respectively (\#\#P $<0.01$ and \#\#\#P $<0.001$ vs. EA + MCAO; $n=$ $5 /$ group, data were expressed as mean \pm SD). 

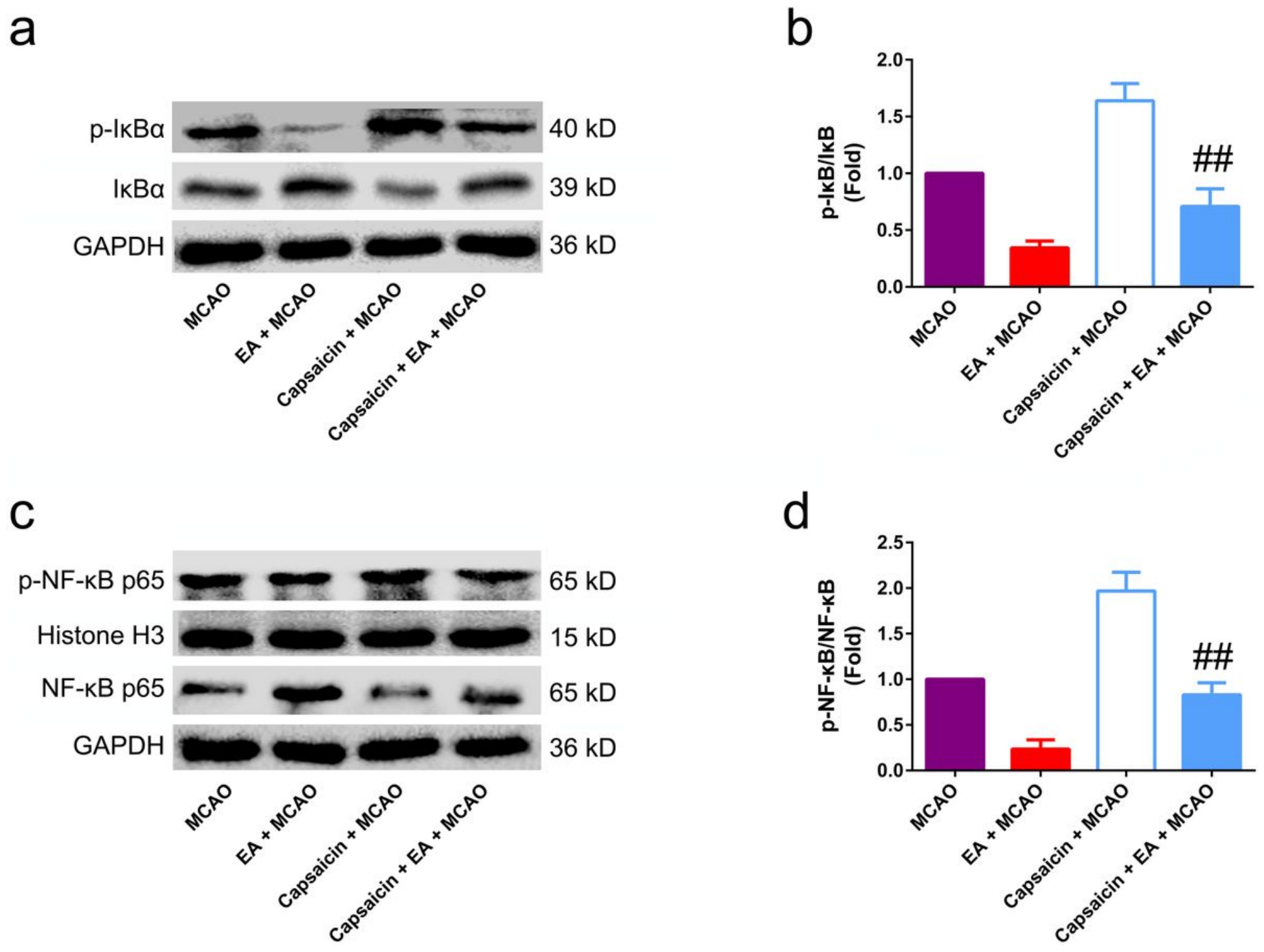

Figure 11

Capsaicin revoked the suppressive effect of EA pretreatment on NF-KB signaling activation in MCAO rats. (a) Cytoplasmic levels of $І \mathrm{kBa}$ and $\mathrm{p}$-IкBa proteins were detected by Western blot. (b) Relative protein expression of $\mathrm{p}-\mathrm{IkBa} / \mathrm{lkBa}$ was statistically analyzed. (c) Nuclear p-NF-kB (p65) and cytoplasmic NF-kB (p65) expression at protein level were detected by Western blot. (d) Relative protein expression of p-NF-KB (p65)/NF-KB (p65) was statistically analyzed (\#\#P<0.01 vs. EA + MCAO; $n=5 /$ group, data were expressed as mean $\pm \mathrm{SD}$ ). 


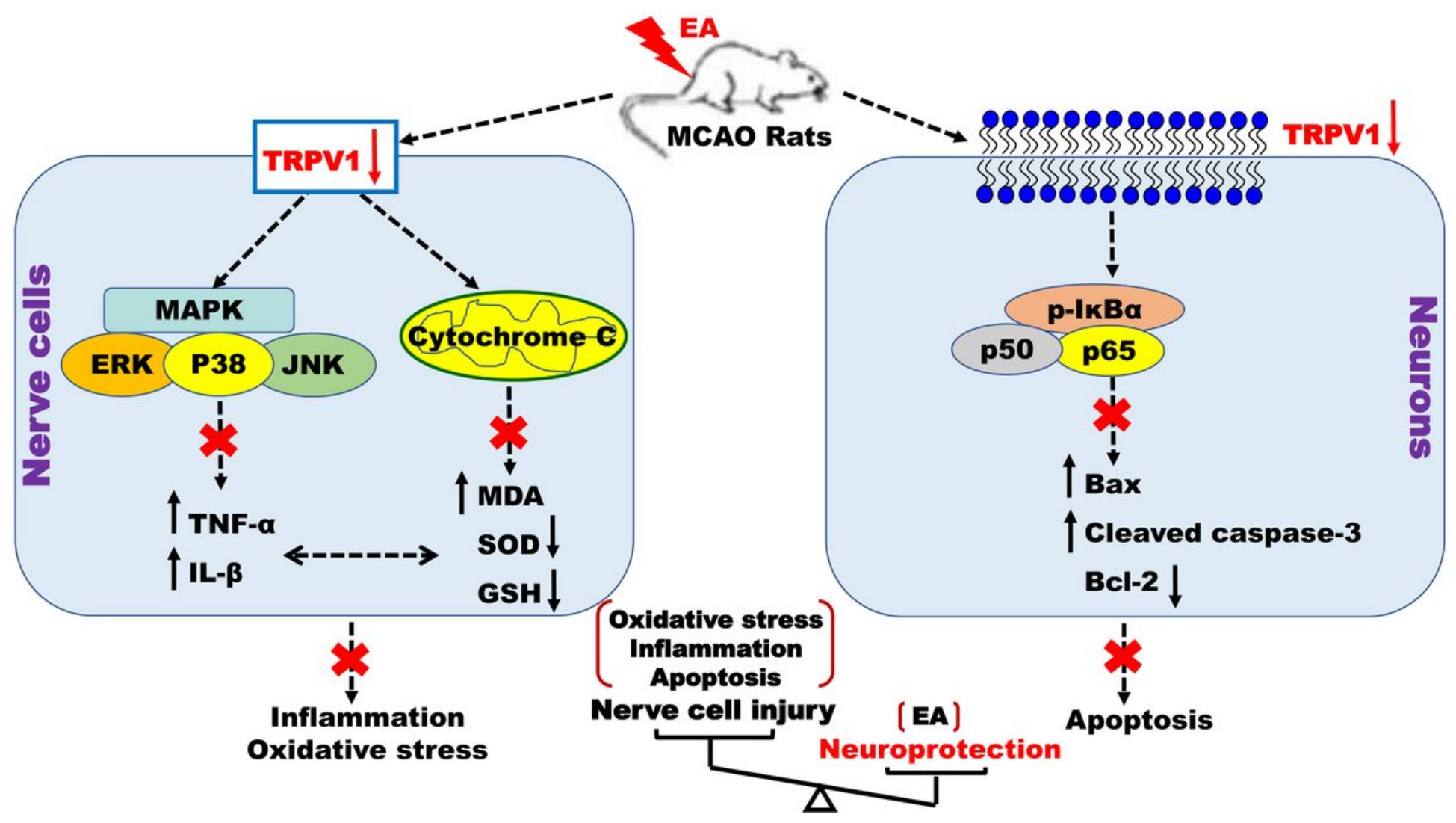

Figure 12

Proposed mechanisms for neuroprotection of EA pretreatment against cerebral ischemia-reperfusion injury in rats. EA pretreatment at GV20, bilateral BL23 and SP6 acupoints decreased TRPV1 expression in hippocampus of the MCAO rat. On the one hand, our previous study reported that downregulation of TRPV1 inhibited CIRI-induced mitochondrial damage and P38 MAPK phosphorylation, resulting in subsequent suppression of oxidative injury and inflammation. On the other hand, accompanying reduction of TRPV1 expression in the current study, EA pretreatment significantly prevented NF-KB transcriptional activity and regulated apoptosis-related protein expression, such as decreasing Bax and cleaved caspase-3 as well as increasing Bcl-2, which contributes to the anti-apoptosis activity of EA pretreatment in MCAO rats. The current findings shed light on the involvement of TRPV1-mediated antiapoptosis in EA pretreatment-evoked neuroprotection against cerebral ischemia-reperfusion injury via inhibiting NF-KB signaling pathway. 